\title{
Wellposedness of second order backward SDEs
}

\section{Journal Article}

Author(s):

Soner, Mete (D; Touzi, Nizar; Zhang, Jianfeng

Publication date:

2012-06

Permanent link:

https://doi.org/10.3929/ethz-b-000049492

\section{Rights / license:}

In Copyright - Non-Commercial Use Permitted

Originally published in:

Probability Theory and Related Fields 153(1-2), https://doi.org/10.1007/s00440-011-0342-y 


\title{
Wellposedness of second order backward SDEs
}

\author{
H. Mete Soner · Nizar Touzi · Jianfeng Zhang
}

Received: 5 April 2010 / Revised: 22 December 2010 / Published online: 4 February 2011 (C) Springer-Verlag 2011

\begin{abstract}
We provide an existence and uniqueness theory for an extension of backward SDEs to the second order. While standard Backward SDEs are naturally connected to semilinear PDEs, our second order extension is connected to fully nonlinear PDEs, as suggested in Cheridito et al. (Commun. Pure Appl. Math. 60(7):1081-1110, 2007). In particular, we provide a fully nonlinear extension of the Feynman-Kac formula. Unlike (Cheridito et al. in Commun. Pure Appl. Math. 60(7):1081-1110, 2007), the alternative formulation of this paper insists that the equation must hold under a non-dominated family of mutually singular probability measures. The key argument is a stochastic representation, suggested by the optimal control interpretation, and analyzed in the accompanying paper (Soner et al. in Dual Formulation of Second Order Target Problems. arXiv:1003.6050, 2009).
\end{abstract}

H. Mete Soner research was partly supported by the European Research Council under the grant 228053-FiRM. Financial support from the ETH Foundation and Swiss Finance Institute are also gratefully acknowledged.

N. Touzi research was supported by the Chair Financial Risks of the Risk Foundation sponsored by Société Générale, the Chair Derivatives of the Future sponsored by the Fédération Bancaire Française, and the Chair Finance and Sustainable Development sponsored by EDF and Calyon.

J. Zhang research was supported in part by NSF grants DMS 06-31366 and DMS 10-08873.

H. M. Soner $(\varangle)$

Departement Mathematik, ETH (Swiss Federal Institute of Technology), Zürich and Swiss Finance Institute, Rämistrasse 101, 8092 Zurich, Switzerland

e-mail: hmsoner@ethz.ch

N. Touzi

CMAP, Ecole Polytechnique Paris, 91128 Palaiseau, France

e-mail: nizar.touzi@polytechnique.edu

J. Zhang

Department of Mathematics, University of Southern California, Los Angeles, CA 90089-2532, USA

e-mail: jianfenz@usc.edu 
Keywords Backward SDEs · Non-dominated family of mutually singular measures Viscosity solutions for second order PDEs

Mathematics Subject Classification (2000) $\quad 60 \mathrm{H} 10 \cdot 60 \mathrm{H} 30$

\section{Introduction}

Backward stochastic differential equations (BSDEs) appeared in Bismut [1] in the linear case, and received considerable attention since the seminal paper of Pardoux and Peng [12]. The various developments are motivated by applications in probabilistic numerical methods for partial differential equations (PDEs), stochastic control, stochastic differential games, theoretical economics and financial mathematics.

On a filtered probability space $\left(\Omega, \mathcal{F},\left\{\mathcal{F}_{t}\right\}_{t \in[0,1]}, \mathbb{P}\right)$ generated by a Brownian motion $W$ with values in $\mathbb{R}^{d}$, a solution to a one-dimensional BSDE consists of a pair of progressively measurable processes $(Y, Z)$ taking values in $\mathbb{R}$ and $\mathbb{R}^{d}$, respectively, such that

$$
Y_{t}=\xi-\int_{t}^{1} f_{S}\left(Y_{s}, Z_{S}\right) d s-\int_{t}^{1} Z_{s} d W_{s}, \quad t \in[0,1], \mathbb{P} \text {-a.s. }
$$

where $f$ is a progressively measurable function from $[0,1] \times \Omega \times \mathbb{R} \times \mathbb{R}^{d}$ to $\mathbb{R}$, and $\xi$ is an $\mathcal{F}_{1}$-measurable random variable.

If the randomness in the parameters $f$ and $\xi$ is induced by the current value of a state process defined by a forward stochastic differential equation (SDE), then the BSDE is referred to as a Markov BSDE and its solution can be written as a deterministic function of time and the current value of the state process. For simplicity, we assume the forward process to be reduced to the Brownian motion, then under suitable regularity assumptions, this function can be shown to be the solution of a parabolic semilinear PDE.

$$
\begin{aligned}
-\partial_{t} v-h^{0}\left(t, x, v, D v, D^{2} v\right) & =0 \\
\text { where } h^{0}(t, x, y, z, \gamma) & :=\frac{1}{2} \operatorname{Tr}[\gamma]-f(t, x, y, z) .
\end{aligned}
$$

In particular, this connection is the main ingredient for the Pardoux and Peng extension of the Feynman-Kac formula to semilinear PDEs. For a larger review of the theory of BSDEs, we refer to El Karoui et al. [8].

Motivated by applications in financial mathematics and probabilistic numerical methods for PDEs, Cheridito et al. [4] introduced the notion of Second Order BSDEs (2BSDEs). The key issue is that, in the Markov case studied by [4], 2BSDEs are connected to the larger class of fully nonlinear PDEs. This is achieved by introducing a further dependence of the generator $f$ on a process $\gamma$ which essentially identifies to the Hessian of the solution of the corresponding PDE. Then, a uniqueness result is proved in an appropriate set $\mathcal{Z}$ for the process $Z$. The linear 2BSDE example reported 
in Sect. 7.1 below shows clearly that the specification of the class $\mathcal{Z}$ is crucial, and can not recover the natural class of square integrable processes, as in classical BSDEs. However, except for the trivial case where the PDE has a sufficiently smooth solution, the existence problem was left open in [4].

In this paper, we provide a complete theory of existence and uniqueness for 2BSDEs. The key idea is a slightly different definition of 2BSDEs which consists in reinforcing the condition that the $2 \mathrm{BSDE}$ must hold $\mathbb{P}$-a.s. for every probability measure $\mathbb{P}$ in a non-dominated class of mutually singular measures introduced in Sect. 2 below. The precise definition is reported in Sect. 3. This new point of view is inspired from the quasi-sure analysis of Denis and Martini [6] who established the connection between the so-called hedging problem in uncertain volatility models and the so-called BlackScholes-Barrenblatt PDE. The latter is fully nonlinear and has a simple piecewise linear dependence on the second order term. We also observe an intimate connection between [6] and the $G$-stochastic integration theory of Peng [13], see Denis et al. [7], and our paper [16].

In the present framework, uniqueness follows from a stochastic representation suggested by the optimal control interpretation. Our construction follows the idea of Peng [13]. When the terminal random variable $\xi$ is in the space $\operatorname{UC}_{b}(\Omega)$ of bounded uniformly continuous maps of $\omega$, the former stochastic representation is shown in our accompanying paper [17] to be the solution of the 2BSDE. Then, we define the closure of $\mathrm{UC}_{b}(\Omega)$ under an appropriate norm. Our main result then shows that for any terminal random variable in this closure, the solution of the 2BSDE can be obtained as a limit of a sequence of solutions corresponding to bounded uniformly continuous final datum $\left(\xi_{n}\right)_{n}$. These are the main results of this paper and are reported in Sect. 4.

Finally, we explore in Sects. 5 and 6 the connection with fully nonlinear PDEs. In particular, we prove a fully nonlinear extension of the Feynman-Kac stochastic representation formula. Moreover, under some conditions, we show that the solution of a Markov 2BSDE is a deterministic function of the time and the current state which is a viscosity solution of the corresponding fully nonlinear PDE.

\section{Preliminaries}

Let $\Omega:=\left\{\omega \in C\left([0,1], \mathbb{R}^{d}\right): \omega_{0}=0\right\}$ be the canonical space equipped with the uniform norm $\|\omega\|_{\infty}:=\sup _{0 \leq t \leq 1}\left|\omega_{t}\right|, B$ the canonical process, $\mathbb{P}_{0}$ the Wiener measure, $\mathbb{F}:=\left\{\mathcal{F}_{t}\right\}_{0 \leq t \leq 1}$ the filtration generated by $B$, and $\mathbb{F}^{+}:=\left\{\mathcal{F}_{t}^{+}, 0 \leq t \leq 1\right\}$ the right limit of $\mathbb{F}$.

\subsection{The local martingale measures}

We say a probability measure $\mathbb{P}$ is a local martingale measure if the canonical process $B$ is a local martingale under $\mathbb{P}$. By Föllmer [9] (see also Karandikar [10] for a more general result), there exists an $\mathbb{F}$-progressively measurable process, denoted as $\int_{0}^{t} B_{s} d B_{s}$, which coincides with the Itô's integral, $\mathbb{P}$-a.s. for all local martingale measures $\mathbb{P}$. In particular, this provides a pathwise definition of 


$$
\langle B\rangle_{t}:=B_{t} B_{t}^{\mathrm{T}}-2 \int_{0}^{t} B_{s} d B_{s}^{\mathrm{T}} \quad \text { and } \quad \hat{a}_{t}:=\varlimsup_{\varepsilon \downarrow 0} \frac{1}{\varepsilon}\left(\langle B\rangle_{t}-\langle B\rangle_{t-\varepsilon}\right),
$$

where ${ }^{\mathrm{T}}$ denotes the transposition, and the $\varlimsup$ im is componentwise. Clearly, $\langle B\rangle$ coincides with the $\mathbb{P}$-quadratic variation of $B, \mathbb{P}$-a.s. for all local martingale measures $\mathbb{P}$.

Let $\overline{\mathcal{P}}_{W}$ denote the set of all local martingale measures $\mathbb{P}$ such that

$$
\langle B\rangle_{t} \text { is absolutely continuous in } t \text { and } \hat{a} \text { takes values in } \mathbb{S}_{d}^{>0}, \quad \mathbb{P} \text {-a.s. }
$$

where $\mathbb{S}_{d}^{>0}$ denotes the space of all $d \times d$ real valued positive definite matrices. We note that, for different $\mathbb{P}_{1}, \mathbb{P}_{2} \in \overline{\mathcal{P}}_{W}$, in general $\mathbb{P}_{1}$ and $\mathbb{P}_{2}$ are mutually singular. This is illustrated by the following example.

Example 2.1 Let $d=1, \mathbb{P}_{1}:=\mathbb{P}_{0} \circ(\sqrt{2} B)^{-1}$, and $\Omega_{i}:=\left\{\langle B\rangle_{t}=(1+i) t, t \geq\right.$ $0\}, i=0$, 1 . Then, $\mathbb{P}_{0}, \mathbb{P}_{1} \in \overline{\mathcal{P}}_{W}, \mathbb{P}_{0}\left(\Omega_{0}\right)=\mathbb{P}_{1}\left(\Omega_{1}\right)=1$, and $\mathbb{P}_{0}\left(\Omega_{1}\right)=\mathbb{P}_{1}\left(\Omega_{0}\right)=0$. That is, $\mathbb{P}_{0}$ and $\mathbb{P}_{1}$ are mutually singular.

For any $\mathbb{P} \in \overline{\mathcal{P}}_{W}$, it follows from the Lévy characterization that the Itô's stochastic integral under $\mathbb{P}$

$$
W_{t}^{\mathbb{P}}:=\int_{0}^{t} \hat{a}_{s}^{-1 / 2} d B_{s}, \quad t \in[0,1], \quad \mathbb{P} \text {-a.s. }
$$

defines a $\mathbb{P}$-Brownian motion.

This paper concentrates on the subclass $\overline{\mathcal{P}}_{S} \subset \overline{\mathcal{P}}_{W}$ consisting of all probability measures

$$
\mathbb{P}^{\alpha}:=\mathbb{P}_{0} \circ\left(X^{\alpha}\right)^{-1} \text { where } X_{t}^{\alpha}:=\int_{0}^{t} \alpha_{s}^{1 / 2} d B_{s}, t \in[0,1], \mathbb{P}_{0} \text {-a.s. }
$$

for some $\mathbb{F}$-progressively measurable process $\alpha$ taking values in $\mathbb{S}_{d}^{>0}$ with $\int_{0}^{1}\left|\alpha_{t}\right| d t<$ $\infty, \mathbb{P}_{0}$-a.s. With $\overline{\mathbb{F}}^{\mathbb{P}}$ (resp. $\overline{\mathbb{F}^{\mathbb{P}^{\mathbb{P}}}}$ ) denoting the $\mathbb{P}$-augmentation of the right-limit filtration generated by $B$ (resp. by $W^{\mathbb{P}}$ ), we recall from [15] that

$$
\overline{\mathcal{P}}_{S}=\left\{\mathbb{P} \in \overline{\mathcal{P}}_{W}: \overline{\mathbb{F}^{\mathbb{P}^{\mathbb{P}}}}=\overline{\mathbb{F}}^{\mathbb{P}}\right\},
$$

and every $\mathbb{P} \in \overline{\mathcal{P}}_{S}$ satisfies the Blumenthal zero-one law and the martingale representation property.

Remark 2.2 Let the process $\alpha$ be as above. Then by Lemma 2.2 in [17],

- there exists an $\mathbb{F}$-progressively measurable mapping $\beta_{\alpha}$ such that $B_{t}=\beta_{\alpha}\left(t, X^{\alpha}\right)$, $t \leq 1, \mathbb{P}_{0}$-a.s. 
- the quadratic variation of the canonical process under $\mathbb{P}^{\alpha}$ is characterized by $\hat{a}(B)=$ $\alpha \circ \beta_{\alpha}(B), d t \times \mathbb{P}^{\alpha}$-a.s.

Remark 2.3 As a consequence of the latter remark, given process $a$ with values in $\mathbb{S}_{d}^{>0}$ and $\int_{0}^{1}\left|a_{t}\right| d t<\infty$, it is not clear whether there exists a process $\alpha$ as above so that the canonical process $\hat{a}=a, \mathbb{P}^{\alpha}$-a.s. The answer to this subtle question is negative in general, as shown by the example

$$
\alpha_{t}:=\mathbf{1}_{[0,2]}\left(\hat{a}_{t}\right)+3 \mathbf{1}_{(2, \infty)}\left(\hat{a}_{t}\right), \quad t \in[0,1] .
$$

This will raise some technical problems in Sect. 5.2.

Remark 2.4 Let $\mathbb{P} \in \overline{\mathcal{P}}_{S}$ be fixed. It follows from the Blumenthal zero-one law that $\mathbb{E}^{\mathbb{P}}\left[\xi \mid \mathcal{F}_{t}\right]=\mathbb{E}^{\mathbb{P}}\left[\xi \mid \mathcal{F}_{t}^{+}\right], \mathbb{P}$-a.s. for any $t \in[0,1]$ and $\mathbb{P}$-integrable $\xi$. In particular, this shows that any $\mathcal{F}_{t}^{+}$-measurable random variable has an $\mathcal{F}_{t}$-measurable $\mathbb{P}$-modification.

\subsection{The nonlinear generator}

Our nonlinear generator is a map

$$
H_{t}(\omega, y, z, \gamma):[0,1] \times \Omega \times \mathbb{R} \times \mathbb{R}^{d} \times D_{H} \rightarrow \mathbb{R}
$$

where $D_{H} \subset \mathbb{R}^{d \times d}$ is a given subset containing 0 . The corresponding conjugate of $H$ with respect to $\gamma$ takes values in $\mathbb{R} \cup\{\infty\}$ and is given by:

$$
\begin{aligned}
F_{t}(\omega, y, z, a) & :=\sup _{\gamma \in D_{H}}\left\{\frac{1}{2} a: \gamma-H_{t}(\omega, y, z, \gamma)\right\}, \quad a \in \mathbb{S}_{d}^{>0} ; \\
\hat{F}_{t}(y, z) & :=F_{t}\left(y, z, \hat{a}_{t}\right) \text { and } \hat{F}_{t}^{0}:=\hat{F}_{t}(0,0) .
\end{aligned}
$$

Here and in the sequel $a: \gamma$ denotes the trace of the product matrix $a \gamma$.

We denote by $D_{F_{t}(y, z)}$ the domain of $F$ in $a$ for fixed $(t, \omega, y, z)$.

Example 2.5 The following are some examples of nonlinearities:

1) Let $H_{t}(y, z, \gamma):=\frac{1}{2} a^{0}: \gamma$ for some matrix $a^{0} \in \mathbb{S}_{d}^{>0}$. Here $D_{H}=\mathbb{S}_{d}$, and we directly calculate that $F_{t}\left(\omega, y, z, a^{0}\right)=0$ and $F_{t}(y, z, a)=\infty$ whenever $a_{t}(\omega) \neq a^{0}$. So $D_{F_{t}(y, z)}=\left\{a^{0}\right\}$.

2) A more interesting nonlinearity considered by Peng [13] will be commented later and is defined by $H_{t}(y, z, \gamma):=\frac{1}{2} \sup _{a \in[a, \bar{a}]}(a: \gamma)$. Here again $D_{H}=\mathbb{S}_{d}$, and we directly compute that $F_{t}(\omega, y, z, a)=0$ for $a \in[\underline{a}, \bar{a}]$, and $\infty$ otherwise. Hence $D_{F_{t}(y, z)}=[\underline{a}, \bar{a}]$.

3) Our last example is motivated by the problem of hedging under gamma constraints in financial mathematics. In the one-dimensional case, given two scalar $\underline{\Gamma}<0<\bar{\Gamma}$, the nonlinearity is $H_{t}(y, z, \gamma)=\frac{1}{2} \gamma$ for $\gamma \in[\underline{\Gamma}, \bar{\Gamma}]$, and $\infty$ otherwise. Here, $D_{H}=[\underline{\Gamma}, \bar{\Gamma}]$ and $F_{t}(\omega, y, z, a)=\frac{1}{2}\left[\bar{\Gamma}(a-1)^{+}-\underline{\Gamma}(a-1)^{-}\right]$. In this example $D_{F_{t}(y, z)}=\mathbb{R}$. 
For the reason explained in Remark 2.9 below, in this paper we shall fix a constant $\kappa$ :

$$
1<\kappa \leq 2
$$

and restrict the probability measures in the following subset $\mathcal{P}_{H}^{\kappa} \subset \overline{\mathcal{P}}_{S}$ :

Definition 2.6 Let $\mathcal{P}_{H}^{\kappa}$ denote the collection of all those $\mathbb{P} \in \overline{\mathcal{P}}_{S}$ such that

$$
\begin{aligned}
& \underline{a}_{\mathbb{P}} \leq \hat{a} \leq \bar{a}_{\mathbb{P}}, \quad d t \times d \mathbb{P} \text {-a.s. for some } \underline{a}_{\mathbb{P}}, \bar{a}_{\mathbb{P}} \in \mathbb{S}_{d}^{>0}, \\
& \quad \text { and } \mathbb{E}^{\mathbb{P}}\left[\left(\int_{0}^{1}\left|\hat{F}_{t}^{0}\right|^{\kappa} d t\right)^{\frac{2}{\kappa}}\right]<\infty .
\end{aligned}
$$

It is clear that $\mathcal{P}_{H}^{\kappa}$ is decreasing in $\kappa$, and $\hat{a}_{t} \in D_{F_{t}(0,0)}, d t \times d \mathbb{P}$-a.s. for all $\mathbb{P} \in \mathcal{P}_{H}^{\kappa}$. Also, we emphasize on the fact that the bounds $\left(\underline{a}_{\mathbb{P}}, \bar{a}_{\mathbb{P}}\right)$ are not uniform in $\mathbb{P}$. In fact this restriction on the set of measure is not essential. For instance, if the nonlinearity (and the terminal data introduced later on) are bounded, then the bound is not needed.

Definition 2.7 We say a property holds $\mathcal{P}_{H}^{\kappa}$-quasi-surely ( $\mathcal{P}_{H}^{\kappa}$-q.s. for short) if it holds $\mathbb{P}$-a.s. for all $\mathbb{P} \in \mathcal{P}_{H}^{\kappa}$.

Throughout this paper, the nonlinearity is assumed to satisfy the following conditions.

Assumption 2.8 $\mathcal{P}_{H}^{\kappa}$ is not empty, and the domain $D_{F_{t}(y, z)}=D_{F_{t}}$ is independent of $(\omega, y, z)$. Moreover, in $D_{F_{t}}, F$ is $\mathbb{F}$-progressively measurable, uniformly continuous in $\omega$ under the uniform convergence norm, and

$$
\left|\hat{F}_{t}(y, z)-\hat{F}_{t}\left(y^{\prime}, z^{\prime}\right)\right| \leq C\left(\left|y-y^{\prime}\right|+\left|\hat{a}^{1 / 2}\left(z-z^{\prime}\right)\right|\right), \quad \mathcal{P}_{H}^{\kappa} \text {-q.s. }
$$

for all $t \in[0,1], y, y^{\prime} \in \mathbb{R}, z, z^{\prime} \in \mathbb{R}^{d}$.

Clearly, one can formulate conditions on $H$ which imply the above Assumption. We prefer to place our assumptions on $F$ directly because this function will be the main object for our subsequent analysis.

\subsection{The spaces and norms}

We now introduce the spaces and norms which will be needed for the formulation of the second order BSDEs. Notice that all subsequent notations extend to the case $\kappa=1$.

For $p \geq 1, L_{H}^{p, \kappa}$ denotes the space of all $\mathcal{F}_{1}$-measurable scalar r.v. $\xi$ with

$$
\|\xi\|_{L_{H}^{p, \kappa}}^{p}:=\sup _{\mathbb{P}_{\mathcal{P}} \mathcal{P}_{H}^{\kappa}} \mathbb{E}^{\mathbb{P}}\left[|\xi|^{p}\right]<\infty
$$


$\mathbb{H}_{H}^{p, \kappa}$ denotes the space of all $\mathbb{F}^{+}$-progressively measurable $\mathbb{R}^{d}$-valued processes $Z$ with

$$
\|Z\|_{\mathbb{H}_{H}^{p, \kappa}}^{p}:=\sup _{\mathbb{P}_{\mathcal{P}}^{\kappa}} \mathbb{E}^{\mathbb{P}}\left[\left(\int_{0}^{1}\left|\hat{a}_{t}^{1 / 2} Z_{t}\right|^{2} d t\right)^{p / 2}\right]<\infty
$$

$\mathbb{D}_{H}^{p, \kappa}$ denotes the space of all $\mathbb{F}^{+}$-progressively measurable $\mathbb{R}$-valued processes $Y$ with

$$
\mathcal{P}_{H}^{\kappa} \text {-q.s. càdlàg paths, and }\|Y\|_{\mathbb{D}_{H}^{p, \kappa}}^{p}:=\sup _{\mathbb{P}_{\mathcal{P}}^{\kappa}} \mathbb{E}^{\mathbb{P}}\left[\sup _{0 \leq t \leq 1}\left|Y_{t}\right|^{p}\right]<\infty \text {. }
$$

For each $\xi \in L_{H}^{1, \kappa}, \mathbb{P} \in \mathcal{P}_{H}^{\kappa}$, and $t \in[0,1]$, denote

$$
\mathbb{E}_{t}^{H, \mathbb{P}}[\xi]:=\operatorname{ess}_{\mathbb{P}^{\prime} \in \mathcal{P}_{H}^{\kappa}(t+, \mathbb{P})} \mathbb{P} \mathbb{E}_{t}^{\mathbb{P}^{\prime}}[\xi] \text { where } \mathcal{P}_{H}^{\kappa}(t+, \mathbb{P}):=\left\{\mathbb{P}^{\prime} \in \mathcal{P}_{H}^{\kappa}: \mathbb{P}^{\prime}=\mathbb{P} \text { on } \mathcal{F}_{t}^{+}\right\}
$$

It follows from Remark 2.4 that $\mathbb{E}_{t}^{\mathbb{P}}[\xi]:=\mathbb{E}^{\mathbb{P}}\left[\xi \mid \mathcal{F}_{t}\right]=\mathbb{E}^{\mathbb{P}}\left[\xi \mid \mathcal{F}_{t}^{+}\right]$, $\mathbb{P}$-a.s. Then, for each $p \geq \kappa$, we define

$$
\begin{aligned}
\mathbb{L}_{H}^{p, \kappa} & :=\left\{\xi \in L_{H}^{p, \kappa}:\|\xi\|_{\left.\mathbb{L}_{H}^{p, \kappa}<\infty\right\} \text { where }}\right. \\
\|\xi\|_{\mathbb{L}_{H}^{p, \kappa}}^{p} & :=\sup _{\mathbb{P} \in \mathcal{P}_{H}^{\kappa}} \mathbb{E}^{\mathbb{P}}\left[\underset{0 \leq t \leq 1}{\operatorname{ess} \sup _{0}}\left(\mathbb{E}_{t}^{H, \mathbb{P}}\left[|\xi|^{\kappa}\right]\right)^{p / \kappa}\right] .
\end{aligned}
$$

The norm $\|\cdot\|_{\mathbb{L}_{H}^{p, \kappa}}$ is somewhat less standard. Below, we justify this definition.

Remark 2.9 Assume $\mathcal{P}_{H}:=\mathcal{P}_{H}^{\kappa}$ and $L_{H}^{p}:=L_{H}^{p, \kappa}$ do not depend on $\kappa$ (e.g. when $\hat{F}^{0}$ is bounded).

(i) For $1 \leq \kappa_{1} \leq \kappa_{2} \leq p$, it is clear that

$$
\|\xi\|_{L_{H}^{p}} \leq\|\xi\|_{\mathbb{L}_{H}^{p, \kappa_{1}}} \leq\|\xi\|_{\mathbb{L}_{H}^{p, \kappa_{2}}} \text { and thus } \mathbb{L}_{H}^{p, \kappa_{2}} \subset \mathbb{L}_{H}^{p, \kappa_{1}} \subset L_{H}^{p}
$$

Moreover, as in our paper [16] Lemma 6.2, under certain technical conditions, we have

$$
\|\xi\|_{\mathbb{L}_{H}^{p_{1}, p_{1}}} \leq C_{p_{2} / p_{1}}\|\xi\|_{L_{H}^{p_{2}}} \text { and thus } \quad L_{H}^{p_{2}} \subset \mathbb{L}_{H}^{p_{1}, p_{1}} \text {, for any } 1 \leq p_{1}<p_{2}
$$

(ii) In our paper [16], we used the norm $\|\cdot\|_{\mathbb{L}_{H}^{p, 1}}$. However, this norm does not work in the present paper due to the presence of the nonlinear generator, see Lemma 4.2. So in this paper we shall assume $\kappa>1$ in order to obtain the norm estimates. 
(iii) In the classical case where $\mathcal{P}_{H}$ is reduced to a single measure $\mathcal{P}_{H}=\left\{\mathbb{P}_{0}\right\}$, we have $\mathbb{E}_{t}^{H, \mathbb{P}_{0}}=\mathbb{E}_{t}^{\mathbb{P}_{0}}$ and the process $\left\{\mathbb{E}_{t}^{H, \mathbb{P}_{0}}\left[|\xi|^{\kappa}\right], t \in[0,1]\right\}$ is a $\mathbb{P}_{0}$-martingale, then it follows immediately from the Doob's maximal inequality that, for all $1 \leq \kappa<p$,

$\|\xi\|_{L^{p}\left(\mathbb{P}_{0}\right)}=\|\xi\|_{L_{H}^{p}} \leq\|\xi\|_{\mathbb{L}_{H}^{p, \kappa}} \leq C_{p, \kappa}\|\xi\|_{L_{H}^{p}}$ and thus $\mathbb{L}_{H}^{p, \kappa}=L_{H}^{p}=L^{p}\left(\mathbb{P}_{0}\right)$.

However, the above equivalence does not hold when $\kappa=p$.

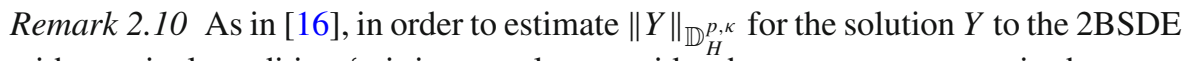
with terminal condition $\xi$, it is natural to consider the supremum over $t$ in the norm of $\xi$. In fact we can show that the process $M_{t}:=\mathbb{E}_{t}^{H, \mathbb{P}}\left[|\xi|^{\kappa}\right]$ a $\mathbb{P}$-supermartingale. Therefore it admits a càdlàg version and thus the term $\sup _{t \in[0,1]} M_{t}$ is measurable.

Finally, we denote by $\mathrm{UC}_{b}(\Omega)$ the collection of all bounded and uniformly continuous maps $\xi: \Omega \longrightarrow \mathbb{R}$ with respect to the $\|\cdot\|_{\infty}$-norm, and we let

$$
\mathcal{L}_{H}^{p, \kappa}:=\text { the closure of } \mathrm{UC}_{b}(\Omega) \text { under the norm }\|\cdot\|_{\mathbb{L}_{H}^{p, \kappa}} \text {, for every } 1 \leq \kappa \leq p .
$$

Similar to (2.11), we have

Remark 2.11 In the case $\mathcal{P}_{H}^{\kappa}=\left\{\mathbb{P}_{0}\right\}$, we have $\mathcal{L}_{H}^{p, \kappa}=\mathbb{L}_{H}^{p, \kappa}=L_{H}^{p, \kappa}=L^{p}\left(\mathbb{P}_{0}\right)$ for $1 \leq \kappa<p$.

\section{The second order BSDEs}

We shall consider the following second order BSDE (2BSDE for short):

$$
Y_{t}=\xi-\int_{t}^{1} \hat{F}_{s}\left(Y_{s}, Z_{s}\right) d s-\int_{t}^{1} Z_{s} d B_{s}+K_{1}-K_{t}, \quad 0 \leq t \leq 1, \quad \mathcal{P}_{H}^{\kappa} \text {-q.s. }
$$

Definition 3.1 For $\xi \in \mathbb{L}_{H}^{2, \kappa}$, we say $(Y, Z) \in \mathbb{D}_{H}^{2, \kappa} \times \mathbb{H}_{H}^{2, \kappa}$ is a solution to 2BSDE (3.1) if

- $Y_{T}=\xi, \mathcal{P}_{H}^{\kappa}$-q.s.

- For each $\mathbb{P} \in \mathcal{P}_{H}^{\kappa}$, the process $K^{\mathbb{P}}$ defined below has nondecreasing paths, $\mathbb{P}$-a.s.:

$$
K_{t}^{\mathbb{P}}:=Y_{0}-Y_{t}+\int_{0}^{t} \hat{F}_{s}\left(Y_{s}, Z_{s}\right) d s+\int_{0}^{t} Z_{s} d B_{s}, \quad 0 \leq t \leq 1, \quad \mathbb{P} \text {-a.s. }
$$


- The family $\left\{K^{\mathbb{P}}, \mathbb{P} \in \mathcal{P}_{H}^{\kappa}\right\}$ defined in (3.2) satisfies the following minimum condition:

$$
K_{t}^{\mathbb{P}}=\underset{\mathbb{P}^{\prime} \in \mathcal{P}_{H}^{\kappa}(t+, \mathbb{P})}{\operatorname{ess} \inf _{t}^{\mathbb{P}}} \mathbb{E}_{t}^{\mathbb{P}^{\prime}}\left[K_{1}^{\mathbb{P}^{\prime}}\right], \quad \mathbb{P} \text {-a.s. for all } \mathbb{P} \in \mathcal{P}_{H}^{\kappa}, t \in[0,1]
$$

Moreover, if the family $\left\{K^{\mathbb{P}}, \mathbb{P} \in \mathcal{P}_{H}^{\kappa}\right\}$ can be aggregated into a universal process $K$, we call $(Y, Z, K)$ a solution of $2 \operatorname{BSDE}(3.1)$.

Clearly, we may rewrite (3.2) as

$$
Y_{t}=\xi-\int_{t}^{1} \hat{F}_{s}\left(Y_{s}, Z_{s}\right) d s-\int_{t}^{1} Z_{s} d B_{s}+K_{1}^{\mathbb{P}}-K_{t}^{\mathbb{P}}, \quad 0 \leq t \leq 1, \quad \mathbb{P} \text {-a.s. }
$$

In particular, if $(Y, Z, K)$ is a solution of $2 \mathrm{BSDE}$ (3.1) in the sense of the above definition, then it satisfies (3.1) $\mathcal{P}_{H}^{\kappa}$-q.s. $\mathbb{P}^{\prime}$-a.s.

Finally, we note that, if $\mathbb{P}^{\prime} \in \mathcal{P}_{H}^{\kappa}(t+, \mathbb{P})$, then $K_{s}^{\mathbb{P}}=K_{s}^{\mathbb{P}^{\prime}}, 0 \leq s \leq t, \mathbb{P}$-a.s. and

\subsection{Connection with the second order stochastic target problem [17]}

Let $(Y, Z)$ be a solution of 2BSDE (3.1). If the conjugate in (2.6) has measurable maximizer, that is, there exists a process $\Gamma$ such that

$$
\frac{1}{2} \hat{a}_{t}: \Gamma_{t}-H_{t}\left(Y_{t}, Z_{t}, \Gamma_{t}\right)=\hat{F}_{t}\left(Y_{t}, Z_{t}\right),
$$

then $(Y, Z, \Gamma)$ satisfies

$$
\begin{aligned}
Y_{t}= & \xi-\int_{t}^{1}\left[\frac{1}{2} \hat{a}_{s}: \Gamma_{s}-H_{s}\left(Y_{s}, Z_{s}, \Gamma_{s}\right)\right] d s \\
& -\int_{t}^{1} Z_{s} d B_{s}+K_{1}-K_{t}, 0 \leq t \leq 1, \mathcal{P}_{H}^{\kappa} \text {-q.s. }
\end{aligned}
$$

If $Z$ is a semi-martingale under each $\mathbb{P} \in \mathcal{P}$ and $d\langle Z, B\rangle_{t}=\Gamma_{t} d\langle B\rangle_{t}, \mathcal{P}_{H}^{\kappa}$-q.s., then,

$$
Y_{t}=\xi+\int_{t}^{1} H_{S}\left(Y_{s}, Z_{S}, \Gamma_{s}\right) d s-\int_{t}^{1} Z_{s} \circ d B_{s}+K_{1}-K_{t}, \quad 0 \leq t \leq 1, \quad \mathcal{P}_{H}^{\kappa} \text {-q.s. }
$$

Here o denotes the Stratonovich integral. We note that (3.7), (3.6), and (3.1) correspond to the second order target problem which was first introduced in [14] under a slightly 
different formulation. The present form, together with its first and second relaxations, were introduced in [17]. In particular, in the Markovian case, the process $\Gamma$ essentially corresponds to the second order derivative of the solution to a fully nonlinear PDE, see Sect. 5. This justifies the denomination as "Second Order" BSDE of [4]. We choose to define 2BSDE in the form of (3.1), rather than (3.6) or (3.7), because this formulation is most appropriate for establishing the wellposedness result, which is the main result of this paper and will be reported in Sect. 4 below.

\subsection{An alternative formulation of 2BSDEs}

In [4], the authors investigate the following so called 2BSDE in Markovian framework:

$$
\left\{\begin{array}{l}
Y_{t}=g\left(B_{1}\right)+\int_{t}^{1} h\left(s, B_{s}, Y_{s}, Z_{s}, \Gamma_{s}\right) d s-\int_{t}^{1} Z_{s} \circ d B_{s}, \quad 0 \leq t \leq 1, \quad \mathbb{P}_{0} \text {-a.s. } \\
d Z_{t}=\Gamma_{t} d B_{t}+A_{t} d t,
\end{array}\right.
$$

where $h$ is a deterministic function. Then uniqueness is proved in an appropriate space $\mathcal{Z}$ for $Z$. The specification of $\mathcal{Z}$ is crucial, and there can be no uniqueness result if the solution is allowed to be a general square integrable process. Indeed, the following "simplest" 2BSDE with $d=1$ has multiple solutions in the natural square integrable space:

$$
\left\{\begin{array}{l}
Y_{t}=\int_{t}^{1} \frac{1}{2} c \Gamma_{s} d s-\int_{t}^{1} Z_{s} \circ d B_{s}, \quad 0 \leq t \leq 1, \quad \mathbb{P}_{0} \text {-a.s. } \\
d Z_{t}=\Gamma_{t} d B_{t}+A_{t} d t
\end{array}\right.
$$

where $c \neq 1$ is a constant. See Example 7.1 below. The reason is that, unless $c=1, \mathbb{P}_{0}$ is not in $\mathcal{P}_{H}^{\kappa}$ for $H(\gamma):=\frac{1}{2} c \gamma$. Also see Sect. 3.4 below.

\subsection{Connection with $G$-expectations and $G$-martingales}

In [16] we established the martingale representation theorem for $G$-martingales, which were introduced by Peng [13]. In our framework, this corresponds to the specification $H_{t}(y, z, \gamma)=G(\gamma):=\frac{1}{2} \sup _{\underline{a} \leq a \leq \bar{a}}(a: \gamma)$, for some $\underline{a}, \bar{a} \in \mathbb{S}_{d}^{>0}$.

As an extension of [16], and as a special case of our current setting, we set

$$
H_{t}(y, z, \gamma):=G(\gamma)-f_{t}(y, z)
$$

Then one can easily check that:

- $D_{F_{t}}=[\underline{a}, \bar{a}]$ and $F_{t}(y, z, a)=f_{t}(y, z)$ for all $a \in[\underline{a}, \bar{a}]$; 
- $\mathcal{P}_{H}^{\kappa}=\left\{\mathbb{P} \in \overline{\mathcal{P}}_{s}: \underline{a} \leq \hat{a} \leq \bar{a}, d t \times d \mathbb{P}\right.$-a.s. and $\left.\mathbb{E}^{\mathbb{P}}\left[\left(\int_{0}^{1}\left|f_{t}(0,0)\right|^{\kappa} d t\right)^{\frac{2}{\kappa}}\right]<\infty\right\}$.

In this case (3.1) is reduced to the following 2BSDE:

$$
Y_{t}=\xi+\int_{t}^{1} f_{s}\left(Y_{s}, Z_{s}\right) d s-\int_{t}^{1} Z_{s} d B_{s}+K_{1}-K_{t}, \quad \mathcal{P}_{H}^{\kappa} \text {-q.s. }
$$

Moreover, we may decompose $K$ into $d K_{t}=k_{t} d t+d K_{t}^{0}$, where $k \geq 0$ and $d K_{t}^{0}$ is a measure singular to the Lebesgue measure $d t$. One can easily check that there exists process $\Gamma$ such that $G\left(\Gamma_{t}\right)-\frac{1}{2} \hat{a}_{t}: \Gamma_{t}=k_{t}$. Then (3.11) becomes

$$
\begin{aligned}
Y_{t}= & \xi+\int_{t}^{1}\left(\frac{1}{2} \hat{a}_{s}: \Gamma_{s}-G\left(\Gamma_{s}\right)+f_{s}\left(Y_{s}, Z_{s}\right)\right) d s \\
& -\int_{t}^{1} Z_{s} d B_{s}+K_{1}^{0}-K_{t}^{0}, \quad \mathcal{P}_{H}^{\kappa} \text {-q.s. }
\end{aligned}
$$

The wellposedness of the latter $G$-BSDE (with $K^{0}=0$ and $\kappa=2$ ) was left by Peng as an open problem. We remark that, although the above two forms are equivalent, we prefer (3.11) than (3.12) because the component $\Gamma$ of the solution is not unique, and we have no appropriate norm for the process $\Gamma$.

\subsection{Connection with the standard BSDE}

Let $H$ be the following linear function of $\gamma$ :

$$
H_{t}(y, z, \gamma)=\frac{1}{2} I_{d}: \gamma-f_{t}(y, z)
$$

where $I_{d}$ is the identity matrix in $\mathbb{R}^{d}$. We remark that in this case we do not need to assume that $f$ is uniformly continuous in $\omega$. Then, under obvious extension of notations, we have

$$
D_{F_{t}(\omega)}=\left\{I_{d}\right\} \text { and } \hat{F}_{t}(y, z)=f_{t}(y, z)
$$

Assume that $\mathbb{E}^{\mathbb{P}_{0}}\left[\int_{0}^{1}\left|f_{t}(0,0)\right|^{2} d t\right]<\infty$, then $\mathcal{P}_{H}^{\kappa}=\mathcal{P}_{H}^{2}=\left\{\mathbb{P}_{0}\right\}$. In this case, the minimum condition (3.3) implies

$$
0=K_{0}=\mathbb{E}^{\mathbb{P}_{0}}\left[K_{1}\right] \text { and thus } K=0, \quad \mathbb{P}_{0} \text {-a.s. }
$$


Hence, the 2BSDE (3.1) is equivalent to the following standard BSDE:

$$
Y_{t}=\xi-\int_{t}^{1} f_{s}\left(Y_{s}, Z_{s}\right) d s-\int_{t}^{1} Z_{s} d B_{S}, \quad 0 \leq t \leq 1, \quad \mathbb{P}_{0} \text {-a.s. }
$$

We note that, by Remark 2.11 , in this case we have

$$
\mathcal{L}_{H}^{2, \kappa}=\mathbb{L}_{H}^{2, \kappa}=L_{H}^{2, \kappa}=\mathbb{L}^{2}\left(\mathbb{P}_{0}\right) \text { for all } 1 \leq \kappa<2
$$

\section{Wellposedness of 2BSDEs}

Throughout this paper Assumption 2.8 and the following assumption will always be in force.

Assumption 4.1 The process $\hat{F}^{0}$ satisfies the integrability condition:

$$
\phi_{H}^{2, \kappa}:=\sup _{\mathbb{P}_{\mathcal{P}}^{\kappa}} \mathbb{E}^{\mathbb{P}}\left[\underset{0 \leq t \leq 1}{\operatorname{ess} \sup }\left(\mathbb{E}_{t}^{H, \mathbb{P}}\left[\int_{0}^{1}\left|\hat{F}_{s}^{0}\right|^{\kappa} d s\right]\right)^{\frac{2}{\kappa}}\right]<\infty .
$$

Clearly the definition of $\phi_{H}^{2, \kappa}$ above is motivated by the norm $\|\xi\|_{\mathbb{L}_{H}^{2, \kappa}}$ in (2.10), and it satisfies

$$
\sup _{\mathbb{P} \in \mathcal{P}_{H}^{\kappa}} \mathbb{E}^{\mathbb{P}}\left[\left(\int_{0}^{1}\left|\hat{F}_{t}^{0}\right| d t\right)^{2}\right] \leq \phi_{H}^{2, \kappa}
$$

For any $\mathbb{P} \in \mathcal{P}_{H}^{\kappa}, \mathbb{F}^{+}$-stopping time $\tau$, and $\mathcal{F}_{\tau}^{+}$-measurable random variable $\xi \in$ $\mathbb{L}^{2}(\mathbb{P})$, let $\left(\mathcal{Y}^{\mathbb{P}}, \mathcal{Z}^{\mathbb{P}}\right):=\left(\mathcal{Y}^{\mathbb{P}}(\tau, \xi), \mathcal{Z}^{\mathbb{P}}(\tau, \xi)\right)$ denote the solution to the following standard BSDE:

$$
\mathcal{Y}_{t}^{\mathbb{P}}=\xi-\int_{t}^{\tau} \hat{F}_{s}\left(\mathcal{Y}_{s}^{\mathbb{P}}, \mathcal{Z}_{s}^{\mathbb{P}}\right) d s-\int_{t}^{\tau} \mathcal{Z}_{s}^{\mathbb{P}} d B_{s}, \quad 0 \leq t \leq \tau, \mathbb{P} \text {-a.s. }
$$

We have the following result which is slightly stronger than the standard ones in the literature. The proof is provided in Sect. 7.2 of the Appendix for completeness. 
Lemma 4.2 Suppose Assumption 2.8 holds. Then, for each $\mathbb{P} \in \mathcal{P}_{H}^{\kappa}$, the BSDE (4.3) has a unique solution satisfying the following estimates:

$$
\begin{aligned}
& \left|\mathcal{Y}_{t}^{\mathbb{P}}\right|^{2} \leq C_{\kappa}\left(\mathbb{E}_{t}^{\mathbb{P}}\left[|\xi|^{\kappa}+\int_{t}^{1}\left|\hat{F}_{s}^{0}\right|^{\kappa} d s\right]\right)^{\frac{2}{\kappa}}, \quad 0 \leq t \leq 1, \mathbb{P} \text {-a.s. } \\
& \mathbb{E}^{\mathbb{P}}\left[\int_{0}^{1}\left|\hat{a}_{t}^{1 / 2} \mathcal{Z}_{t}^{\mathbb{P}}\right|^{2} d t\right] \leq C_{\kappa} \mathbb{E}^{\mathbb{P}}\left[\sup _{0 \leq t \leq 1}\left(\mathbb{E}_{t}^{\mathbb{P}}\left[|\xi|^{\kappa}+\int_{0}^{1}\left|\hat{F}_{s}^{0}\right|^{\kappa} d s\right]\right)^{\frac{2}{\kappa}}\right] .
\end{aligned}
$$

We note that in above lemma, and in all subsequent results, we shall denote by $C$ a generic constant which may vary from line to line and depends only on the dimension $d$ and the Lipschitz constant in (2.9) of Assumption 2.8. We shall also denote by $C_{\kappa}$ a generic constant which may depend on $\kappa$ as well. We emphasize that, due to the Lipschitz condition (2.9), the constants $C$ and $C_{\kappa}$ in the estimates will not depend on the bounds $\underline{a}_{\mathbb{P}}$ and $\bar{a}_{\mathbb{P}}$ in (2.8).

\subsection{Representation and uniqueness of the solution}

Theorem 4.3 Let Assumptions 2.8 and 4.1 hold. Assume that $\xi \in \mathbb{L}_{H}^{2, \kappa}$ and that $(Y, Z) \in \mathbb{D}_{H}^{2, \kappa} \times \mathbb{H}_{H}^{2, \kappa}$ is a solution to $2 B S D E$ (3.1). Then, for any $\mathbb{P} \in \mathcal{P}_{H}^{\kappa}$ and $0 \leq t_{1}<t_{2} \leq 1$

$$
Y_{t_{1}}=\underset{\mathbb{P}^{\prime} \in \mathcal{P}_{H}^{\kappa}\left(t_{1}+, \mathbb{P}\right)}{\operatorname{ess} \sup _{t_{1}}} \mathbb{P} \mathcal{P}^{\mathbb{P}^{\prime}}\left(t_{2}, Y_{t_{2}}\right), \quad \mathbb{P} \text {-a.s. }
$$

Consequently, the 2BSDE (3.1) has at most one solution in $\mathbb{D}_{H}^{2, \kappa} \times \mathbb{H}_{H}^{2, \kappa}$.

Proof. We first prove the last statement about uniqueness. So suppose that (4.6) holds. Then as a special case with $t_{2}=1$ we obtain

$$
Y_{t}=\underset{\mathbb{P}^{\prime} \in \mathcal{P}_{H}^{\kappa}(t+, \mathbb{P})}{\operatorname{ess} \sup _{t} \mathbb{P}} \mathcal{Y}_{t}^{\mathbb{P}^{\prime}}(1, \xi), \quad \mathbb{P} \text {-a.s. for all } \mathbb{P} \in \mathcal{P}_{H}^{\kappa}, t \in[0,1]
$$

Therefore $Y$ is unique. To prove the uniqueness of $Z$, we observe that $d\langle Y, B\rangle_{t}=$ $Z_{t} d\langle B\rangle_{t}, \mathcal{P}_{H}^{\kappa}$-q.s.. Therefore the uniqueness of $Y$ implies that $Z$ is also unique.

It remains to prove (4.6).

(i) Fix $0 \leq t_{1}<t_{2} \leq 1$ and $\mathbb{P} \in \mathcal{P}_{H}^{\kappa}$. For any $\mathbb{P}^{\prime} \in \mathcal{P}_{H}^{\kappa}\left(t_{1}+, \mathbb{P}\right)$, note that

$$
Y_{t}=Y_{t_{2}}-\int_{t}^{t_{2}} \hat{F}_{s}\left(Y_{s}, Z_{s}\right) d s-\int_{t}^{1} Z_{s} d B_{s}+K_{t_{2}}^{\mathbb{P}^{\prime}}-K_{t}^{\mathbb{P}^{\prime}}, \quad 0 \leq t \leq t_{2}, \quad \mathbb{P}^{\prime} \text {-a.s. }
$$


and that $K^{\mathbb{P}^{\prime}}$ is nondecreasing, $\mathbb{P}^{\prime}$-a.s. By (2.9), and applying the comparison principle for standard BSDE under $\mathbb{P}$, we have $Y_{t_{1}} \geq \mathcal{Y}_{t_{1}}^{\mathbb{P}^{\prime}}\left(t_{2}, Y_{t_{2}}\right), \mathbb{P}^{\prime}$-a.s. Since $\mathbb{P}^{\prime}=\mathbb{P}$ on $\mathcal{F}_{t_{1}}^{+}$, we get $Y_{t_{1}} \geq \mathcal{Y}_{t_{1}}^{\mathbb{P}^{\prime}}\left(t_{2}, Y_{t_{2}}\right), \mathbb{P}$-a.s. and thus

$$
Y_{t_{1}} \geq \underset{\mathbb{P}^{\prime} \in \mathcal{P}_{H}^{\kappa}\left(t_{1}+, \mathbb{P}\right)}{\operatorname{ess} \operatorname{Yup}_{t_{1}}} \mathbb{P}^{\mathbb{P}^{\prime}}\left(t_{2}, Y_{t_{2}}\right), \quad \mathbb{P} \text {-a.s. }
$$

(ii) We now prove the other direction of the inequality. Fix $\mathbb{P} \in \mathcal{P}_{H}^{\kappa}$. For every $\mathbb{P}^{\prime} \in$ $\mathcal{P}_{H}^{\kappa}\left(t_{1}+, \mathbb{P}\right)$, denote:

$$
\delta Y:=Y-\mathcal{Y}^{\mathbb{P}^{\prime}}\left(t_{2}, Y_{t_{2}}\right) \text { and } \delta Z:=Z-\mathcal{Z}^{\mathbb{P}^{\prime}}\left(t_{2}, Y_{t_{2}}\right)
$$

By the Lipschitz conditions (2.9), there exist bounded processes $\lambda, \eta$ such that

$$
\delta Y_{t}=\int_{t}^{t_{2}}\left(\lambda_{s} \delta Y_{s}+\eta_{s} \hat{a}_{s}^{1 / 2} \delta Z_{s}\right) d s-\int_{t}^{t_{2}} \delta Z_{s} d B_{s}+K_{t_{2}}^{\mathbb{P}^{\prime}}-K_{t}^{\mathbb{P}^{\prime}}, \quad t \leq t_{2}, \mathbb{P}^{\prime} \text {-a.s. }
$$

Define:

$$
M_{t}:=\exp \left(-\int_{0}^{t} \eta_{s} \hat{a}_{s}^{-1 / 2} d B_{s}-\int_{0}^{t}\left(\lambda_{s}+\frac{1}{2}\left|\eta_{s}\right|^{2}\right) d s\right), \quad 0 \leq t \leq t_{2}, \quad \mathbb{P}^{\prime} \text {-a.s. }
$$

By Itô's formula, we have:

$$
d\left(M_{t} \delta Y_{t}\right)=M_{t}\left(\delta Z_{t}-\delta Y_{t} \eta_{t} \hat{a}_{t}^{-1 / 2}\right) d B_{t}-M_{t} d K_{t}^{\mathbb{P}^{\prime}}, \quad t_{1} \leq t \leq t_{2}, \mathbb{P}^{\prime} \text {-a.s. }
$$

Then, since $\delta Y_{t_{2}}=0$, using standard localization arguments if necessary, we compute that:

$$
\begin{aligned}
Y_{t_{1}}-\mathcal{Y}_{t_{1}}^{\mathbb{P}^{\prime}}\left(t_{2}, Y_{t_{2}}\right) & =\delta Y_{t_{1}}=M_{t_{1}}^{-1} \mathbb{E}_{t_{1}}^{\mathbb{P}^{\prime}}\left[\int_{t_{1}}^{t_{2}} M_{t} d K_{t}^{\mathbb{P}^{\prime}}\right] \\
& \leq \mathbb{E}_{t_{1}}^{\mathbb{P}^{\prime}}\left[\sup _{t_{1} \leq t \leq t_{2}}\left(M_{t_{1}}^{-1} M_{t}\right)\left(K_{t_{2}}^{\mathbb{P}^{\prime}}-K_{t_{1}}^{\mathbb{P}^{\prime}}\right)\right]
\end{aligned}
$$

by the non-decrease of $K^{\mathbb{P}^{\prime}}$. By the boundedness of $\lambda, \eta$, for every $p \geq 1$ we have, 


$$
\mathbb{E}_{t_{1}}^{\mathbb{P}^{\prime}}\left[\sup _{t_{1} \leq t \leq t_{2}}\left(M_{t_{1}}^{-1} M_{t}\right)^{p}+\sup _{t_{1} \leq t \leq t_{2}}\left(M_{t_{1}} M_{t}^{-1}\right)^{p}\right] \leq C_{p}, \quad t_{1} \leq t \leq t_{2}, \quad \mathbb{P}^{\prime} \text {-a.s. }
$$

Then it follows from the Hölder inequality that:

$$
\begin{aligned}
Y_{t_{1}}-\mathcal{Y}_{t}^{\mathbb{P}^{\prime}}\left(t_{2}, Y_{t_{2}}\right) & \leq\left(\mathbb{E}_{t_{1}}^{\mathbb{P}^{\prime}}\left[\sup _{t_{1} \leq t \leq t_{2}}\left(M_{t_{1}}^{-1} M_{t}\right)^{3}\right]\right)^{1 / 3}\left(\mathbb{E}_{t_{1}}^{\mathbb{P}^{\prime}}\left[\left(K_{t_{2}}^{\mathbb{P}^{\prime}}-K_{t_{1}}^{\mathbb{P}^{\prime}}\right)^{3 / 2}\right]\right)^{2 / 3} \\
& \leq C\left(\mathbb{E}_{t_{1}}^{\mathbb{P}^{\prime}}\left[K_{t_{2}}^{\mathbb{P}^{\prime}}-K_{t_{1}}^{\mathbb{P}^{\prime}}\right] \mathbb{E}_{t_{1}}^{\mathbb{P}^{\prime}}\left[\left(K_{t_{2}}^{\mathbb{P}^{\prime}}-K_{t_{1}}^{\mathbb{P}^{\prime}}\right)^{2}\right]\right)^{1 / 3}
\end{aligned}
$$

We shall prove in Step (iii) below that

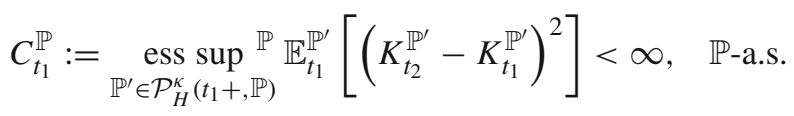

Then, it follows from the last inequality that

$$
\begin{aligned}
& Y_{t_{1}}-\underset{\left.\mathbb{P}^{\prime} \in \mathcal{P}_{H}^{\kappa} \sup _{1}+, \mathbb{P}\right)}{\operatorname{ess} \mathcal{Y}_{t_{1}}^{\mathbb{P}^{\prime}}\left(t_{2}, Y_{t_{2}}\right)} \\
& \leq C\left(C_{t_{1}}^{\mathbb{P}}\right)^{1 / 3} \underset{\mathbb{P}^{\prime} \in \mathcal{P}_{H}^{\kappa}\left(t_{1}+, \mathbb{P}\right)}{\operatorname{ess} \inf _{t_{1}}}\left(\mathbb{E}_{t_{1}}^{\mathbb{P}^{\prime}}\left[K_{t_{2}}^{\mathbb{P}^{\prime}}-K_{t_{1}}^{\mathbb{P}^{\prime}}\right]\right)^{1 / 3}=0, \quad \mathbb{P} \text {-a.s. }
\end{aligned}
$$

by the minimum condition (3.3).

(iii) It remains to show that the estimate (4.13) holds. By the definition of the family $\left\{K^{\mathbb{P}}, \mathbb{P} \in \mathcal{P}_{H}^{\kappa}\right\}$ we have:

$$
\sup _{\mathbb{P}^{\prime} \in \mathcal{P}_{H}^{\kappa}\left(t_{1}+, \mathbb{P}\right)} \mathbb{E}^{\mathbb{P}^{\prime}}\left[\left(K_{t_{2}}^{\mathbb{P}^{\prime}}-K_{t_{1}}^{\mathbb{P}^{\prime}}\right)^{2}\right] \leq C\left(\|Y\|_{\mathbb{D}_{H}^{2, \kappa}}^{2}+\|Z\|_{\mathbb{H}_{H}^{2, \kappa}}^{2}+\phi_{H}^{2, \kappa}\right)<\infty
$$

We next use the definition of the essential supremum, see e.g. Neveu [11] to see that

$$
\underset{\mathbb{P}^{\prime} \in \mathcal{P}_{H}^{\kappa}\left(t_{1}+, \mathbb{P}\right)}{\operatorname{ess} \sup _{t_{1}}} \mathbb{P}^{\mathbb{P}^{\prime}}\left[\left(K_{t_{2}}^{\mathbb{P}^{\prime}}-K_{t_{1}}^{\mathbb{P}^{\prime}}\right)^{2}\right]=\sup _{n \geq 1} \mathbb{E}_{t_{1}}^{\mathbb{P}_{n}}\left[\left(K_{t_{2}}^{\mathbb{P}_{n}}-K_{t_{1}}^{\mathbb{P}_{n}}\right)^{2}\right], \quad \mathbb{P} \text {-a.s. }
$$

for some sequence $\left(\mathbb{P}_{n}\right)_{n \geq 1} \subset \mathcal{P}_{H}^{\kappa}\left(t_{1}+, \mathbb{P}\right)$. Observe that for $\mathbb{P}_{1}^{\prime}, \mathbb{P}_{2}^{\prime} \in \mathcal{P}_{H}^{\kappa}\left(t_{1}+, \mathbb{P}\right)$, there exists $\mathbb{P}^{\prime} \in \mathcal{P}_{H}^{\kappa}\left(t_{1}+, \mathbb{P}\right)$ such that

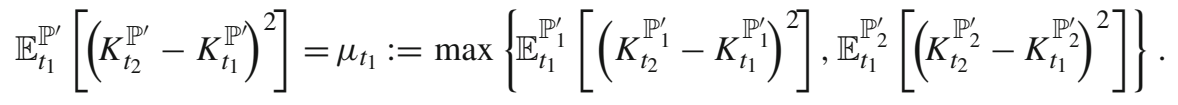


Indeed, set

$$
E_{1}:=\left\{\mu_{t_{1}}=\mathbb{E}_{t_{1}}^{\mathbb{P}_{1}^{\prime}}\left[\left(K_{t_{2}}^{\mathbb{P}_{1}^{\prime}}-K_{t_{1}}^{\mathbb{P}_{1}^{\prime}}\right)^{2}\right]\right\} \quad \text { and } \quad E_{2}:=\Omega \backslash E_{1}
$$

so that both sets are in $\mathcal{F}_{t_{1}}$. We then define the probability measure $\mathbb{P}^{\prime}$ by,

$$
\mathbb{P}^{\prime}[E]:=\mathbb{P}_{1}^{\prime}\left[E \cap E_{1}\right]+\mathbb{P}_{2}^{\prime}\left[E \cap E_{2}\right] \text { for all } E \in \mathcal{F}_{1}
$$

Then, by its definition, $\mathbb{P}^{\prime}$ satisfies (4.16) trivially. Moreover, in Sect. 7.3 of the Appendix, it is proved that

$$
\mathbb{P}^{\prime} \in \mathcal{P}_{H}^{\kappa}\left(t_{1}+, \mathbb{P}\right)
$$

Using this construction, by using a subsequence, if necessary, we rewrite (4.15), as

$$
\underset{\mathbb{P}^{\prime} \in \mathcal{P}_{H}^{\kappa}\left(t_{1}+, \mathbb{P}\right)}{\operatorname{ess} \sup _{t_{1}}} \mathbb{E}_{\mathbb{P}^{\prime}}^{\mathbb{P}_{t_{2}}}\left[\left(K_{t_{1}}^{\mathbb{P}^{\prime}}\right)^{2}\right]=\lim _{n \rightarrow \infty} \uparrow \mathbb{E}_{t_{1}}^{\mathbb{P}_{n}}\left[\left(K_{t_{2}}^{\mathbb{P}_{n}}-K_{t_{1}}^{\mathbb{P}_{n}}\right)^{2}\right]
$$

It follows from (4.14) that

$$
\begin{aligned}
& \left.\left.\mathbb{E}^{\mathbb{P}}\left[\underset{\mathbb{P}^{\prime} \in \mathcal{P}_{H}^{\kappa}\left(t_{1}+, \mathbb{P}\right)}{\operatorname{ess} \sup _{t_{1}}} \mathbb{P}_{\mathbb{P}^{\prime}}^{\mathbb{P}_{t_{2}}}-K_{t_{1}}^{\mathbb{P}^{\prime}}\right)^{2}\right]\right]=\mathbb{E}^{\mathbb{P}}\left[\lim _{n \rightarrow \infty} \uparrow \mathbb{E}_{t_{1}}^{\mathbb{P}_{n}}\left[\left(K_{t_{2}}^{\mathbb{P}_{n}}-K_{t_{1}}^{\mathbb{P}_{n}}\right)^{2}\right]\right] \\
& =\lim _{n \rightarrow \infty} \uparrow \mathbb{E}^{\mathbb{P}_{n}}\left[\left(K_{t_{2}}^{\mathbb{P}_{n}}-K_{t_{1}}^{\mathbb{P}_{n}}\right)^{2}\right] \\
& \leq \sup _{\mathbb{P}^{\prime} \in \mathcal{P}_{H}^{k}\left(t_{1}+, \mathbb{P}\right)} \mathbb{E}^{\mathbb{P}^{\prime}}\left[\left(K_{t_{2}}^{\mathbb{P}^{\prime}}-K_{t_{1}}^{\mathbb{P}^{\prime}}\right)^{2}\right]<\infty
\end{aligned}
$$

by (4.14), which implies the required estimate (4.13).

As an immediate consequence of the representation formula (4.7), together with the comparison principle for BSDEs, we have the following comparison principle for 2BSDEs.

Corollary 4.4 Let Assumptions 2.8 and 4.1 hold. Assume $\xi^{i} \in \mathbb{L}_{H}^{2, \kappa}$ and $\left(Y^{i}, Z^{i}\right) \in$ $\mathbb{D}_{H}^{2, \kappa} \times \mathbb{H}_{H}^{2, \kappa}$ is a corresponding solution of the $2 B S D E(3.1), i=1$, 2. If $\xi^{1} \leq \xi^{2}, \mathcal{P}_{H^{-}}^{\kappa}$ q.s. then $Y^{1} \leq Y^{2}, \mathcal{P}_{H}^{\kappa}$-q.s.

4.2 A priori estimates and the existence of a solution

Theorem 4.5 Let Assumptions 2.8 and 4.1 hold. 
(i) Assume that $\xi \in \mathbb{L}_{H}^{2, \kappa}$ and that $(Y, Z) \in \mathbb{D}_{H}^{2, \kappa} \times \mathbb{H}_{H}^{2, \kappa}$ is a solution to $2 B S D E$ (3.1). Then there exist a constant $C_{\kappa}$ such that

$$
\|Y\|_{\mathbb{D}_{H}^{2, \kappa}}^{2}+\|Z\|_{\mathbb{H}_{H}^{2, \kappa}}^{2}+\sup _{\mathbb{P} \in \mathcal{P}_{H}^{\kappa}} \mathbb{E}^{\mathbb{P}}\left[\left|K_{1}^{\mathbb{P}}\right|^{2}\right] \leq C_{\kappa}\left(\|\xi\|_{\mathbb{L}_{H}^{2, \kappa}}^{2}+\phi_{H}^{2, \kappa}\right)
$$

(ii) Assume that $\xi^{i} \in \mathbb{L}_{H}^{2, \kappa}$ and that $\left(Y^{i}, Z^{i}\right) \in \mathbb{D}_{H}^{2, \kappa} \times \mathbb{H}_{H}^{2, \kappa}$ is a corresponding solution to $2 B S D E$ (3.1), $i=1$, 2. Denote $\delta \xi:=\xi^{1}-\xi^{2}, \delta Y:=Y^{1}-Y^{2}, \delta Z:=$ $Z^{1}-Z^{2}$, and $\delta K^{\mathbb{P}}:=K^{1, \mathbb{P}}-K^{2, \mathbb{P}}$. Then there exists a constant $C_{\kappa}$ such that

$$
\begin{aligned}
& \|\delta Y\|_{\mathbb{D}_{H}^{2, \kappa}} \leq C_{\kappa}\|\delta \xi\|_{\mathbb{L}_{H}^{2, \kappa}}, \\
& \|\delta Z\|_{\mathbb{H}_{H}^{2, \kappa}}^{2}+\sup _{\mathbb{P} \in \mathcal{P}_{H}^{\kappa}} \mathbb{E}^{\mathbb{P}}\left[\sup _{0 \leq t \leq 1}\left|\delta K_{t}^{\mathbb{P}}\right|^{2}\right] \\
& \leq C_{\kappa}\|\delta \xi\|_{\mathbb{L}_{H}^{2, \kappa}}\left(\left\|\xi^{1}\right\|_{\left.\mathbb{L}_{H}^{2, \kappa}+\left\|\xi^{2}\right\|_{\mathbb{L}_{H}^{2, \kappa}}+\left(\phi_{H}^{2, \kappa}\right)^{1 / \mathcal{2}}\right) .}\right.
\end{aligned}
$$

Proof. (i) First, by Lemma 4.2 we have:

$\left|\mathcal{Y}_{t}^{\mathbb{P}}(1, \xi)\right|^{2} \leq C_{\kappa}\left(\mathbb{E}_{t}^{\mathbb{P}}\left[|\xi|^{\kappa}+\int_{t}^{1}\left|\hat{F}_{s}^{0}\right|^{\kappa} d s\right]\right)^{2 / \kappa} \quad, \quad \mathbb{P}$-a.s. for all $\mathbb{P} \in \mathcal{P}_{H}^{\kappa}, t \in[0,1]$

By the representation formula (4.7), this provides

$\left|Y_{t}\right|^{2} \leq C_{\kappa}\left(\mathbb{E}_{t}^{H, \mathbb{P}}\left[|\xi|^{\kappa}+\int_{t}^{1}\left|\hat{F}_{s}^{0}\right|^{\kappa} d s\right]\right)^{2 / \kappa} \quad, \quad \mathbb{P}$-a.s. for all $\mathbb{P} \in \mathcal{P}_{H}^{\kappa}, t \in[0,1]$,

and, by the definition of the norms, we get

$$
\|Y\|_{\mathbb{D}_{H}^{2, \kappa}}^{2} \leq C_{\kappa}\left(\|\xi\|_{\mathbb{L}_{H}^{2, \kappa}}^{2}+\phi_{H}^{2, \kappa}\right)
$$

Next, under each $\mathbb{P} \in \mathcal{P}_{H}^{\kappa}$, applying Itô's formula to $|Y|^{2}$, it follows from the Lipschitz conditions (2.9) that:

$$
\begin{aligned}
\mathbb{E}^{\mathbb{P}}\left[\int_{0}^{1}\left|\hat{a}_{s}^{1 / 2} Z_{s}\right|^{2} d s\right] & \leq \mathbb{E}^{\mathbb{P}}\left[\left|Y_{0}\right|^{2}+\int_{0}^{1}\left|\hat{a}_{s}^{1 / 2} Z_{s}\right|^{2} d s\right] \\
& \leq C \mathbb{E}^{\mathbb{P}}\left[|\xi|^{2}+\int_{0}^{1}\left|Y_{t}\right|\left(\left|\hat{F}_{t}^{0}\right|+\left|Y_{t}\right|+\left|\hat{a}_{t}^{1 / 2} Z_{t}\right|\right) d s+\int_{0}^{1}\left|Y_{t}\right| d K_{t}^{\mathbb{P}}\right]
\end{aligned}
$$




$$
\begin{aligned}
& \leq C \varepsilon^{-1} \mathbb{E}^{\mathbb{P}}\left[|\xi|^{2}+\sup _{0 \leq t \leq 1}\left|Y_{t}\right|^{2}+\left(\int_{0}^{1}\left|\hat{F}_{t}^{0}\right| d t\right)^{2}\right] \\
& +\varepsilon \mathbb{E}^{\mathbb{P}}\left[\int_{0}^{1}\left|\hat{a}_{t}^{1 / 2} Z_{t}\right|^{2} d t+\left|K_{1}^{\mathbb{P}}\right|^{2}\right]
\end{aligned}
$$

for any $\varepsilon \in(0,1]$. By the definition of $K^{\mathbb{P}}$, one gets immediately that

$$
\mathbb{E}^{\mathbb{P}}\left[\left|K_{1}^{\mathbb{P}}\right|^{2}\right] \leq C_{0} \mathbb{E}^{\mathbb{P}}\left[|\xi|^{2}+\sup _{0 \leq t \leq 1}\left|Y_{t}\right|^{2}+\int_{0}^{1}\left|\hat{a}_{t}^{1 / 2} Z_{t}\right|^{2} d t+\left(\int_{0}^{1}\left|\hat{F}_{t}^{0}\right| d t\right)^{2}\right],
$$

for some constant $C_{0}$ independent of $\varepsilon$. Then,

$$
\begin{aligned}
\mathbb{E}^{\mathbb{P}}\left[\int_{0}^{1}\left|\hat{a}_{s}^{1 / 2} Z_{s}\right|^{2} d s\right] \leq & C \varepsilon^{-1} \mathbb{E}^{\mathbb{P}}\left[|\xi|^{2}+\sup _{0 \leq t \leq 1}\left|Y_{t}\right|^{2}+\left(\int_{0}^{1}\left|\hat{F}_{t}^{0}\right| d t\right)^{2}\right] \\
& +\left(1+C_{0}\right) \varepsilon \mathbb{E}^{\mathbb{P}}\left[\int_{0}^{1}\left|\hat{a}_{t}^{1 / 2} Z_{t}\right|^{2} d t\right]
\end{aligned}
$$

where we recall that the constant $C$ changes from line to line. By setting $\varepsilon:=[2(1+$ $\left.\left.C_{0}\right)\right]^{-1}$, this provides

$$
\mathbb{E}^{\mathbb{P}}\left[\int_{0}^{1}\left|\hat{a}_{s}^{1 / 2} Z_{s}\right|^{2} d s\right] \leq C \mathbb{E}^{\mathbb{P}}\left[|\xi|^{2}+\sup _{0 \leq t \leq 1}\left|Y_{t}\right|^{2}+\left(\int_{0}^{1}\left|\hat{F}_{t}^{0}\right| d t\right)^{2}\right] .
$$

By (4.20) and noting that $\phi_{H}^{2,1} \leq \phi_{H}^{2, \kappa}$ for $\kappa>1$, we have

$$
\|Z\|_{\mathbb{H}_{H}^{2, \kappa}}^{2} \leq C\left(\|\xi\|_{\mathbb{L}_{H}^{2, \kappa}}^{2}+\phi_{H}^{2, \kappa}\right) .
$$

This, together with (4.20) and (4.21), proves (4.18).

(ii) First, following the same arguments as in Lemma 4.2, we have

$$
\left|\mathcal{Y}_{t}^{\mathbb{P}}\left(1, \xi_{1}\right)-\mathcal{Y}_{t}^{\mathbb{P}}\left(1, \xi_{2}\right)\right| \leq C\left(\mathbb{E}_{t}^{\mathbb{P}}\left[|\delta \xi|^{\kappa}\right]\right)^{2 / \kappa}, \quad \mathbb{P} \text {-a.s. for all } \mathbb{P} \in \mathcal{P}_{H}^{\kappa}, t \in[0,1]
$$

Then, following similar arguments as in (i) we have

$$
\|\delta Y\|_{\mathbb{D}_{H}^{2, \kappa}} \leq C\|\delta \xi\|_{\mathbb{L}_{H}^{2, \kappa}}
$$


Next, under each $\mathbb{P} \in \mathcal{P}_{H}^{\kappa}$, applying Itô's formula to $|\delta Y|^{2}$ we get

$$
\begin{aligned}
\mathbb{E}^{\mathbb{P}}\left[\int_{0}^{1}\left|\hat{a}_{s}^{1 / 2} \delta Z_{s}\right|^{2} d s\right] \leq & \mathbb{E}^{\mathbb{P}}\left[\left|\delta Y_{0}\right|^{2}+\int_{0}^{1}\left|\hat{a}_{s}^{1 / 2} \delta Z_{s}\right|^{2} d s\right] \\
\leq & C \mathbb{E}^{\mathbb{P}}\left[|\delta \xi|^{2}+\int_{0}^{1}\left|\delta Y_{t}\right|\left(\left|\delta Y_{t}\right|+\left|\hat{a}_{t}^{1 / 2} \delta Z_{t}\right|\right) d s+\left|\int_{0}^{1} \delta Y_{t} d\left(\delta K_{t}^{\mathbb{P}}\right)\right|\right] \\
\leq & C \mathbb{E}^{\mathbb{P}}\left[|\delta \xi|^{2}+\sup _{0 \leq t \leq 1}\left|\delta Y_{t}\right|^{2}+\sup _{0 \leq t \leq 1}\left|\delta Y_{t}\right|\left[K_{1}^{1, \mathbb{P}}+K_{1}^{2, \mathbb{P}}\right]\right] \\
& +\frac{1}{2} \mathbb{E}^{\mathbb{P}}\left[\int_{0}^{1}\left|\hat{a}_{t}^{1 / 2} \delta Z_{t}\right|^{2} d t\right] .
\end{aligned}
$$

Then, by (4.23) and (4.18),

$$
\begin{aligned}
\mathbb{E}^{\mathbb{P}}\left[\int_{0}^{1}\left|\hat{a}_{S}^{1 / 2} \delta Z_{s}\right|^{2} d s\right] & \leq C_{\kappa}\|\delta \xi\|_{\mathbb{L}_{H}^{2, \kappa}}^{2}+C_{\kappa}\|\delta \xi\|_{\mathbb{L}_{H}^{2, \kappa}}\left(\mathbb{E}^{\mathbb{P}}\left[\left|K_{1}^{1, \mathbb{P}}\right|^{2}+\left|K_{1}^{2, \mathbb{P}}\right|^{2}\right]\right)^{1 / 2} \\
& \leq C_{\kappa}\|\delta \xi\|_{\mathbb{L}_{H}^{2, \kappa}}^{2}+C_{\kappa}\|\delta \xi\|_{\mathbb{L}_{H}^{2, \kappa}}\left(\left\|\xi^{1}\right\|_{\mathbb{L}_{H}^{2, \kappa}}+\left\|\xi^{2}\right\|_{\mathbb{L}_{H}^{2, \kappa}}+\left(\phi_{H}^{2, \kappa}\right)^{1 / 2}\right) .
\end{aligned}
$$

The estimate for $\delta K^{\mathbb{P}}$ is obvious now.

We are now ready to state the main result of this paper. Recall that $\mathcal{L}_{H}^{2, \kappa}$ is the closure of $\mathrm{UC}_{b}(\Omega)$ under the norm $\|\cdot\|_{\mathbb{L}_{H}^{2, \kappa}}$.

Theorem 4.6 Let Assumptions 2.8 and 4.1 hold. Then for any $\xi \in \mathcal{L}_{H}^{2, \kappa}$, the $2 B S D E$ (3.1) has a unique solution $(Y, Z) \in \mathbb{D}_{H}^{2, \kappa} \times \mathbb{H}_{H}^{2, \kappa}$.

Proof. (i) We first assume $\xi \in \mathrm{UC}_{b}(\Omega)$. In this case, by Step 2 of the proof of Theorem 4.5 in [17], there exist $(Y, Z) \in \mathbb{D}_{H}^{2, \kappa} \times \mathbb{H}_{H}^{2, \kappa}$ such that $Y_{1}=\xi, \mathcal{P}_{H}^{\kappa}$-q.s. and the $K^{\mathbb{P}}$

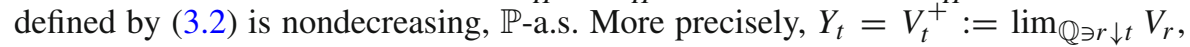
where $V$ is defined in that paper. We notice that the modification of the space of measure $\mathcal{P}_{H}^{\kappa}$ does not alter the arguments. Moreover, by Proposition 4.10 in [17], the representation (4.7) holds:

$$
Y_{t}=\operatorname{ess}_{\mathbb{P}^{\prime} \in \mathcal{P}_{H}^{\kappa}\left(t+, \mathbb{P}^{\prime}\right)} \mathbb{P} \mathcal{Y}_{t}^{\mathbb{P}^{\prime}}(1, \xi), \quad \mathbb{P} \text {-a.s. for all } \mathbb{P} \in \mathcal{P}_{H}^{\kappa}, t \in[0,1]
$$

The construction of $V$ in [17] is crucially based on the so-called regular conditional probability distribution (r.c.p.d., see Sect. 6.1) which allows to define the process $Y$ on $\Omega$ without exception of any zero measure set. Then, $Y$ is shown to satisfy a dynamic programming principle which induces the required decomposition by an appropriate extension of the Doob-Meyer decomposition. 
(ii) It remains to check the minimum condition (3.3). We follow the arguments in the proof of Theorem 4.3. For $t \in[0,1], \mathbb{P} \in \mathcal{P}_{H}^{\kappa}$, and $\mathbb{P}^{\prime} \in \mathcal{P}_{H}^{\kappa}(t+, \mathbb{P})$, we denote $\delta Y:=Y-\mathcal{Y}^{\mathbb{P}^{\prime}}(1, \xi), \delta Y:=Z-\mathcal{Z}^{\mathbb{P}^{\prime}}(1, \xi)$, and we introduce the process $M$ of (4.10). Then, it follows from the non-decrease of $K^{\mathbb{P}^{\prime}}$ that

$$
\begin{aligned}
& Y_{t}-\mathcal{Y}_{t}^{\mathbb{P}^{\prime}}(1, \xi)=\delta Y_{t}=\mathbb{E}_{t}^{\mathbb{P}^{\prime}}\left[\int_{t}^{1} M_{s} d K_{s}^{\mathbb{P}^{\prime}}\right] \\
& \geq \mathbb{E}_{t}^{\mathbb{P}^{\prime}}\left[\left(\inf _{t \leq s \leq 1} M_{t}^{-1} M_{s}\right)\left(K_{1}^{\mathbb{P}^{\prime}}-K_{t}^{\mathbb{P}^{\prime}}\right)\right] \text {. }
\end{aligned}
$$

On the other hand, by (4.12) and (4.25), we estimate by the Hölder inequality that

$$
\begin{aligned}
\mathbb{E}_{t}^{\mathbb{P}^{\prime}}\left[K_{1}^{\mathbb{P}^{\prime}}-K_{t}^{\mathbb{P}^{\prime}}\right] \\
=\mathbb{E}_{t}^{\mathbb{P}^{\prime}}\left[\left(\inf _{t \leq s \leq 1} M_{t}^{-1} M_{s}\right)^{1 / 3}\left(K_{1}^{\mathbb{P}^{\prime}}-K_{t}^{\mathbb{P}^{\prime}}\right)^{1 / 3}\left(\inf _{t \leq s \leq 1} M_{t}^{-1} M_{s}\right)^{-1 / 3}\left(K_{1}^{\mathbb{P}^{\prime}}-K_{t}^{\mathbb{P}^{\prime}}\right)^{2 / 3}\right] \\
\leq\left(\mathbb{E}_{t}^{\mathbb{P}^{\prime}}\left[\left(\inf _{t \leq s \leq 1} M_{t}^{-1} M_{s}\right)\left(K_{1}^{\mathbb{P}^{\prime}}-K_{t}^{\mathbb{P}^{\prime}}\right)\right]\right. \\
\left.\quad \mathbb{E}_{t}^{\mathbb{P}^{\prime}}\left[\sup _{t \leq s \leq 1} M_{t} M_{s}^{-1}\right] \mathbb{E}_{t}^{\mathbb{P}^{\prime}}\left[\left(K_{1}^{\mathbb{P}^{\prime}}-K_{t}^{\mathbb{P}^{\prime}}\right)^{2}\right]\right)^{1 / 3} \\
\leq C\left(\mathbb{E}_{t}^{\mathbb{P}^{\prime}}\left[\left(K_{1}^{\mathbb{P}^{\prime}}\right)^{2}\right] \mathbb{E}_{t}^{\mathbb{P}^{\prime}}\left[\left(\inf _{t \leq s \leq 1} M_{t}^{-1} M_{s}\right)\left(K_{1}^{\mathbb{P}^{\prime}}-K_{t}^{\mathbb{P}^{\prime}}\right)\right]\right)^{1 / 3} \\
\leq C\left(\mathbb{E}_{t}^{\mathbb{P}^{\prime}}\left[\left(K_{1}^{\mathbb{P}^{\prime}}\right)^{2}\right]\right)^{1 / 3}\left(\delta Y_{t}\right)^{1 / 3} .
\end{aligned}
$$

By following the argument of the proof of Theorem 4.3 (ii) and (iii), we then deduce that the family $\left\{K^{\mathbb{P}}, \mathbb{P} \in \mathcal{P}_{H}^{\kappa}\right\}$ inherits the minimum condition (3.3) from (4.24).

(ii) In general, for $\xi \in \mathcal{L}_{H}^{2, \kappa}$, by the definition of the space $\mathcal{L}_{H}^{2, \kappa}$ there exist $\xi_{n} \in$ $\mathrm{UC}_{b}(\Omega)$ such that $\lim _{n \rightarrow \infty}\left\|\xi_{n}-\xi\right\|_{\mathbb{L}_{H}^{2, \kappa}}=0$. Then it is clear that

$$
\sup _{n \geq 1}\left\|\xi_{n}\right\|_{\mathbb{L}_{H}^{2, \kappa}}<\infty \text { and } \lim _{n, m \rightarrow \infty}\left\|\xi_{n}-\xi_{m}\right\|_{\mathbb{L}_{H}^{2, \kappa}}=0 .
$$

Let $\left(Y^{n}, Z^{n}\right) \in \mathbb{D}_{H}^{2, \kappa} \times \mathbb{H}_{H}^{2, \kappa}$ be the solution to 2BSDE (3.1) with terminal condition $\xi_{n}$, and

$$
K_{t}^{n, \mathbb{P}}:=Y_{0}^{n}-Y_{t}^{n}+\int_{0}^{t} \hat{F}_{s}\left(Y_{s}^{n}, Z_{s}^{n}\right) d s+\int_{0}^{t} Z_{s}^{n} d B_{s}, \quad 0 \leq t \leq 1, \quad \mathbb{P} \text {-a.s. }
$$


By Theorem 4.5 , as $n, m \rightarrow \infty$ we have

$$
\begin{aligned}
& \left\|Y^{n}-Y^{m}\right\|_{\mathbb{D}_{H}^{2, \kappa}}^{2}+\left\|Z^{n}-Z^{m}\right\|_{\mathbb{H}_{H}^{2, \kappa}}^{2}+\sup _{\mathbb{P}_{\in} \mathcal{P}_{H}^{\kappa}} \mathbb{E}^{\mathbb{P}}\left[\sup _{0 \leq t \leq 1}\left|K_{t}^{n, \mathbb{P}}-K_{t}^{m, \mathbb{P}}\right|^{2}\right] \\
& \leq C_{\kappa}\left\|\xi_{n}-\xi_{m}\right\|_{\mathbb{L}_{H}^{2, \kappa}}^{2}+C_{\kappa}\left(\left\|\xi_{n}\right\|_{\mathbb{L}_{H}^{2, \kappa}}+\left\|\xi_{m}\right\|_{\mathbb{L}_{H}^{2, \kappa}}+\left\|\hat{F}^{0}\right\|_{\mathbb{H}_{H}^{2, \kappa}}\right)\left\|\xi_{n}-\xi_{m}\right\|_{\mathbb{L}_{H}^{2, \kappa}} \rightarrow 0 .
\end{aligned}
$$

Then by otherwise choosing a subsequence, we may assume without loss of generality that,

$$
\left\|Y^{n}-Y^{m}\right\|_{\mathbb{D}_{H}^{2, \kappa}}^{2}+\left\|Z^{n}-Z^{m}\right\|_{\mathbb{H}_{H}^{2, \kappa}}^{2}+\sup _{\mathbb{P}_{\mathcal{P}}^{\kappa}} \mathbb{E}^{\mathbb{P}}\left[\sup _{0 \leq t \leq 1}\left|K_{t}^{n, \mathbb{P}}-K_{t}^{m, \mathbb{P}}\right|^{2}\right] \leq 2^{-n},
$$

for all $m \geq n \geq 1$. This implies that, for every $\mathbb{P} \in \mathcal{P}_{H}^{\kappa}$ and $m \geq n \geq 1$,

$\mathbb{P}\left[\sup _{0 \leq t \leq 1}\left[\left|Y_{t}^{n}-Y_{t}^{m}\right|^{2}+\left|K_{t}^{n, \mathbb{P}}-K_{t}^{m, \mathbb{P}}\right|^{2}\right]+\int_{0}^{1}\left|Z_{t}^{n}-Z_{t}^{m}\right|^{2} d t>\frac{1}{n}\right] \leq C n 2^{-n}$

Define

$$
Y:=\varlimsup_{n \rightarrow \infty} Y^{n}, \quad Z:=\varlimsup_{n \rightarrow \infty} Z^{n}, \quad K^{\mathbb{P}}:=\varlimsup_{n \rightarrow \infty} K^{n, \mathbb{P}}
$$

where the $\varlimsup$ fim for $Z$ is taken componentwise. It is clear that $Y, Z, K^{\mathbb{P}}$ are all $\mathbb{F}^{+}$-progressively measurable. By (4.29), it follows from the Borel-Cantelli Lemma that

$$
\begin{aligned}
& \lim _{n \rightarrow \infty}\left[\sup _{0 \leq t \leq 1}\left\{\left|Y_{t}^{n}-Y_{t}\right|^{2}+\left|K_{t}^{n, \mathbb{P}}-K_{t}^{\mathbb{P}}\right|^{2}\right\}+\int_{0}^{1}\left|Z_{t}^{n}-Z_{t}\right|^{2} d t\right]=0, \\
& \mathbb{P} \text {-a.s. for all } \mathbb{P} \in \mathcal{P}_{H}^{\kappa} .
\end{aligned}
$$

Since $Y^{n}, K^{n, \mathbb{P}}$ are càdlàg and $K^{n, \mathbb{P}}$ is nondecreasing, this implies that $Y$ is càdlàg, $\mathcal{P}_{H}^{\kappa}$-q.s. and $K^{\mathbb{P}}$ is càdlàg and nondecreasing, $\mathbb{P}$-a.s. Moreover, for every $\mathbb{P} \in \mathcal{P}_{H}^{\kappa}$ and $n \geq 1$, sending $m \rightarrow \infty$ in (4.28) and applying Fatou's lemma under $\mathbb{P}$, we obtain:

$$
\mathbb{E}^{\mathbb{P}}\left[\sup _{0 \leq t \leq 1}\left\{\left|Y_{t}^{n}-Y_{t}\right|^{2}+\left|K_{t}^{n, \mathbb{P}}-K_{t}^{\mathbb{P}}\right|^{2}\right\}+\int_{0}^{1}\left|Z_{t}^{n}-Z_{t}\right|^{2}\right] \leq 2^{-n}
$$


This implies that

$$
\begin{aligned}
& \left\|Y^{n}-Y\right\|_{\mathbb{D}_{H}^{2, \kappa}}^{2}+\left\|Z^{n}-Z\right\|_{\mathbb{H}_{H}^{2, \kappa}}^{2} \\
& +\sup _{\mathbb{P} \in \mathcal{P}_{H}^{\kappa}} \mathbb{E}^{\mathbb{P}}\left[\sup _{0 \leq t \leq 1}\left|K_{t}^{n, \mathbb{P}}-K_{t}^{\mathbb{P}}\right|^{2}\right] \leq 2^{-n} \rightarrow 0, \text { as } n \rightarrow \infty .
\end{aligned}
$$

Then it is clear that $(Y, Z) \in \mathbb{D}_{H}^{2, \kappa} \times \mathbb{H}_{H}^{2, \kappa}$.

Finally, since $\left(Y^{n}, Z^{n}, K^{n, \mathbb{P}}\right)$ satisfy (3.4) and (4.7), the limit $\left(Y, Z, K^{\mathbb{P}}\right)$ also satisfies (3.4) and (4.7). Then by the proof of Theorem 4.6, the family $\left\{K^{\mathbb{P}}, \mathbb{P} \in \mathcal{P}_{H}^{\kappa}\right\}$ satisfies (3.3). Hence $(Y, Z)$ is a solution to $2 \mathrm{BSDE}$ (3.1).

Remark 4.7 After the completion of this paper, Marcel Nutz pointed out that our solution of the 2BSDE in the present contexts is in fact $\mathbb{F}$-progressively measurable, as a consequence of the uniform continuity in $\omega$ in our setting. See Proposition 4.11 in [17]. However, the $\mathbb{F}^{+}$-progressive measurability developed in this paper seems to be more robust to potential extensions of the spaces.

\section{Connection with fully nonlinear PDEs}

\subsection{The Markovian setup}

In this section we consider the case:

$$
H_{t}(\omega, y, z, \gamma)=h\left(t, B_{t}(\omega), y, z, \gamma\right)
$$

where $h:[0,1] \times \mathbb{R}^{d} \times \mathbb{R} \times \mathbb{R}^{d} \times D_{h} \rightarrow \mathbb{R}$ is a deterministic map. Then the corresponding conjugate and bi-conjugate functions become

$$
\begin{array}{ll}
f(t, x, y, z, a):=\sup _{\gamma \in D_{h}}\left\{\frac{1}{2} a: \gamma-h(t, x, y, z, \gamma)\right\}, \quad a \in \mathbb{S}_{d}^{>0}, \\
\hat{h}(t, x, y, z, \gamma):=\sup _{a \in \mathbb{S}_{d}^{>0}}\left\{\frac{1}{2} a: \gamma-f(t, x, y, z, a)\right\}, \quad \gamma \in \mathbb{R}^{d \times d} .
\end{array}
$$

Notice that $-\infty<\hat{h} \leq h$ and $\hat{h}$ is nondecreasing convex in $\gamma$. Also, $\hat{h}=h$ if and only if $h$ is convex and nondecreasing in $\gamma$.

In the present context, we write $\mathcal{P}_{h}^{\kappa}:=\mathcal{P}_{H}^{\kappa}$. The following is a slight strengthening of Assumption 2.8 to our Markov framework.

Assumption 5.1 $\mathcal{P}_{h}^{\kappa}$ is not empty, the domain $D_{f_{t}}$ of the map $a \longmapsto f(t, x, y, a)$ is independent of $(x, y, z)$. Moreover, on $D_{f_{t}}, f$ is uniformly continuous in $t$, uniformly in $a$, and for some constant $C$ and modulus of continuity $\rho$ with polynomial growth: 


$$
\left|f(t, x, y, z, a)-f\left(t, x^{\prime}, y^{\prime}, z^{\prime}, a\right)\right| \leq \rho\left(\left|x-x^{\prime}\right|\right)+C\left(\left|y-y^{\prime}\right|+\left|a^{1 / 2}\left(z_{1}-z_{2}\right)\right|\right),
$$

for all $t \in[0,1], a \in D_{f_{t}}, x, x^{\prime}, z, z^{\prime} \in \mathbb{R}^{d}, y, y^{\prime} \in \mathbb{R}$.

Next, let $g: \mathbb{R}^{d} \rightarrow \mathbb{R}$ be a Lebesgue measurable function. In this section we shall always consider the 2BSDE (3.1) in this Markovian setting with terminal condition $\xi=g\left(B_{1}\right)$ :

$$
\begin{aligned}
Y_{t}= & g\left(B_{1}\right)-\int_{t}^{1} f\left(s, B_{s}, Y_{s}, Z_{s}, \hat{a}_{s}\right) d s \\
& -\int_{t}^{1} Z_{s} d B_{s}+K_{1}-K_{t}, \quad 0 \leq t \leq 1, \mathcal{P}_{H}^{\kappa} \text {-q.s. }
\end{aligned}
$$

Our main objective is to establish the connection $Y_{t}=v\left(t, B_{t}\right), t \in[0,1], \mathcal{P}_{H}^{\kappa}$-q.s. where $v$ solves, in some sense, the following fully nonlinear PDE:

$$
\left\{\begin{array}{l}
\mathcal{L} v(t, x):=\partial_{t} v(t, x)+\hat{h}\left(t, x, v(t, x), D v(t, x), D^{2} v(t, x)\right)=0, \quad 0 \leq t<1 \\
v(1, x)=g(x)
\end{array}\right.
$$

We remark that the nonlinearity of the above PDE is the nondecreasing and convex envelope $\hat{h}$, not the original $h$. This is illustrated by the following example.

Example 5.2 The problem of hedging under gamma constraints in dimension $d=1$, as formulated by Cheridito et al. [3], leads to the specification

$$
h(t, x, y, z, \gamma):=\frac{1}{2} \gamma \text { if } \gamma \in[\underline{\Gamma}, \bar{\Gamma}] \text {, and } \infty \text { otherwise, }
$$

where $\underline{\Gamma}<0<\bar{\Gamma}$ are given constants. Then, direct calculation leads to

$$
\begin{aligned}
& f(a)=\frac{1}{2}\left(\bar{\Gamma}(a-1)^{+}-\underline{\Gamma}(a-1)^{-}\right), \quad a>0, \\
& \hat{h}(\gamma)=\frac{1}{2}(\gamma \vee \underline{\Gamma}) \text { if } \gamma \leq \bar{\Gamma}, \quad \text { and } \infty \text { otherwise. }
\end{aligned}
$$

We will discuss further this case in Example 5.12 below, in order to obtain the nonlinearity appearing in the PDE characterization of [3] for the superhedging problem under gamma constraints. Indeed, Eq. (5.5) needs to be reformulated in some appropriate sense if $D_{h} \neq \mathbb{S}_{d}$, because then $\hat{h}$ may take infinite values, and the meaning of (5.5) is not clear anymore. This leads typically to a boundary layer and requires 
the interpretation of the equation in the relaxed boundary value sense of viscosity solutions, see, e.g. [5].

\subsection{A nonlinear Feynman-Kac representation formula}

Theorem 5.3 Let Assumption 5.1 hold true. Suppose further that $\hat{h}$ is continuous in its domain, $D_{f}$ is independent of $t$ and is bounded both from above and away from 0. Let $v \in C^{1,2}\left([0,1), \mathbb{R}^{d}\right)$ be a classical solution of $(5.5)$ with $\left\{(v, D v)\left(t, B_{t}\right), t \in\right.$ $[0,1]\} \in \mathbb{D}_{H}^{2, \kappa} \times \mathbb{H}_{H}^{2, \kappa}$. Then:

$Y_{t}:=v\left(t, B_{t}\right), Z_{t}:=D v\left(t, B_{t}\right), K_{t}:=\int_{0}^{t} k_{s} d s$

with $k_{t}:=\hat{h}\left(t, B_{t}, Y_{t}, Z_{t}, \Gamma_{t}\right)-\frac{1}{2} \hat{a}_{t}: \Gamma_{t}+f\left(t, B_{t}, Y_{t}, Z_{t}, \hat{a}_{t}\right)$ and $\Gamma_{t}:=D^{2} v\left(t, B_{t}\right)$

is the unique solution of the $2 B S D E$ (5.4).

Proof. By definition $Y_{1}=g\left(B_{1}\right)$ and (5.4) is verified by immediate application of Itô's formula. It remains to prove the minimum condition:

$$
\underset{\mathbb{P}^{\prime} \in \mathcal{P}_{H}^{\kappa}(t+, \mathbb{P})}{\operatorname{ess} \inf _{t}} \mathbb{E}_{\mathbb{P}^{\prime}}^{\mathbb{P}^{\prime}}\left[\int_{t}^{1} k_{s} d s\right]=0 \text { for all } t \in[0,1], \mathbb{P} \in \mathcal{P}_{H}^{\kappa},
$$

by which we can conclude that $(Y, Z, K)$ is a solution of the $2 \operatorname{BSDE}$ (5.4). Since $g\left(B_{1}\right) \in \mathbb{L}_{H}^{2, \kappa}$, the uniqueness follows from Theorems 4.3 and 4.5 (i).

To prove (5.6), we follow the same argument as in the proof of Lemma 3.1 in [8]. For every $\varepsilon>0$, notice that the set

$$
A^{\varepsilon}:=\left\{a \in D_{f}: \hat{h}\left(t, B_{t}, Y_{t}, Z_{t}, \Gamma_{t}\right) \leq \frac{1}{2} a: \Gamma_{t}-f\left(t, B_{t}, Y_{t}, Z_{t}, a\right)+\varepsilon\right\}
$$

is not empty. Then it follows from a measurable selection argument that there exists a predictable process $a^{\varepsilon}$ taking values in $D_{f}$ such that

$$
\hat{h}\left(t, B_{t}, Y_{t}, Z_{t}, \Gamma_{t}\right) \leq \frac{1}{2} a_{t}^{\varepsilon}: \Gamma_{t}-f\left(t, B_{t}, Y_{t}, Z_{t}, a_{t}^{\varepsilon}\right)+\varepsilon
$$

We note that this in particular implies that $\Gamma_{t} \in D_{\hat{h}}$.

In the remainder of this proof, we show the existence of an $\mathbb{F}$-progressively measurable process $\alpha^{\varepsilon}$ with values in $\mathbb{S}_{d}^{>0}$ and $\int_{0}^{1}\left|\alpha_{s}^{\varepsilon}\right| d s<\infty$ such that, $\mathbb{P}^{\alpha^{\varepsilon}}$-a.s., $\hat{a}$ is in $A^{\varepsilon}$. We recall from Remarks 2.2 and 2.3 that this is not guaranteed in general. Notice that this technical difficulty is inherent to the problem and requires to be addressed even if a maximizer for $\hat{h}$ does exist. 
Let $\mathbb{P}:=\mathbb{P}^{\alpha} \in \mathcal{P}_{H}$ and $t_{0} \in[0,1]$ be fixed. Let

$$
\tau_{0}^{\varepsilon}:=1 \wedge \inf \left\{t \geq t_{0} \mid K_{t} \geq K_{t_{0}}+\varepsilon\right\}
$$

and define:

$\tau_{n+1}^{\varepsilon}:=1 \wedge \inf \left\{t \geq \tau_{n}^{\varepsilon} \mid \hat{h}\left(t, B_{t}, Y_{t}, \Gamma_{t}\right) \geq \frac{1}{2} a_{\tau_{n}^{\varepsilon}}^{\varepsilon}: \Gamma_{t}-f\left(t, B_{t}, Y_{t}, Z_{t}, a_{\tau_{n}^{\varepsilon}}^{\varepsilon}\right)+2 \varepsilon\right\}$,

for $n \geq 0$. Since $K$ is continuous, notice that $\tau_{0}^{\varepsilon}>t_{0}, \mathcal{P}_{H}^{\kappa}$-q.s.. Also, since $B, Y, Z, \Gamma$ are all continuous in $t, \tau_{n}^{\varepsilon}$ are $\mathbb{F}$-stopping times and, for any fixed $\omega$, are uniformly continuous in $t$.

Next, for any fixed $a \in D_{f}$, the function $f(., a)$ is continuous. Also $\hat{h}$ is continuous. Then for $\mathcal{P}_{H}^{\kappa}$-q.s. $\omega \in \Omega$,

$$
\begin{aligned}
& \hat{h}\left(t, B_{t}(\omega), Y_{t}(\omega), Z_{t}(\omega), \Gamma_{t}(\omega)\right)-\frac{1}{2} a_{\tau_{n}^{\varepsilon}}^{\varepsilon}(\omega): \Gamma_{t}(\omega) \\
& \quad+f\left(t, B_{t}(\omega), Y_{t}(\omega), Z_{t}(\omega), a_{\tau_{n}^{\varepsilon}}^{\varepsilon}(\omega)\right)
\end{aligned}
$$

is uniformly continuous in $t$ for $t \in\left[\tau_{n}^{\varepsilon}(\omega), 1\right]$. Then $\tau_{n+1}^{\varepsilon}(\omega)-\tau_{n}^{\varepsilon}(\omega) \geq \delta(\varepsilon, \omega)>0$ whenever $\tau_{n+1}^{\varepsilon}(\omega)<1$, where the constant $\delta(\varepsilon, \omega)$ does not depend on $n$. This implies that $\tau_{n}^{\varepsilon}(\omega)=1$ for $n$ large enough. Applying the arguments in Example 4.5 of [15] on $\left[\tau_{0}^{\varepsilon}, 1\right]$, one can easily see that there exists an $\mathbb{F}$-progressively measurable process $\alpha^{\varepsilon}$ taking values in $D_{f}$ such that

$$
\begin{gathered}
\alpha_{t}^{\varepsilon}=\alpha_{t} \text { for } t \in\left[0, \tau_{0}^{\varepsilon}\right] \text { and } \hat{a}_{t}=\sum_{n=0}^{\infty} a_{\tau_{n}^{\varepsilon}}^{\varepsilon} \mathbf{1}_{\left[\tau_{n}^{\varepsilon}, \tau_{n+1}^{\varepsilon}\right)}(t), \\
d t \times d \mathbb{P}^{\alpha^{\varepsilon}} \text {-a.s. on }\left[\tau_{0}^{\varepsilon}, 1\right] \times \Omega .
\end{gathered}
$$

This implies that

$$
\begin{gathered}
\hat{h}\left(t, B_{t}, Y_{t}, Z_{t}, \Gamma_{t}\right) \leq \frac{1}{2} \hat{a}_{t}: \Gamma_{t}-f\left(t, B_{t}, Y_{t}, Z_{t}, \hat{a}_{t}\right)+2 \varepsilon, \\
d t \times d \mathbb{P}^{\alpha^{\varepsilon}} \text {-a.s. on }\left[\tau_{0}^{\varepsilon}, 1\right] \times \Omega,
\end{gathered}
$$

Under our conditions it is obvious that $\mathbb{P}^{\alpha^{\varepsilon}} \in \mathcal{P}_{H}^{\kappa}$, then $\mathbb{P}^{\alpha^{\varepsilon}} \in \mathcal{P}_{H}^{\kappa}\left(t_{0}+, \mathbb{P}\right)$ since $\tau_{0}^{\varepsilon}>t_{0}$. Therefore,

$$
\left.\underset{\mathbb{P}^{\prime} \in \mathcal{P}_{H}^{\kappa}\left(t_{0}+, \mathbb{P}\right)}{\operatorname{ess} \inf _{t_{0}}} \mathbb{P} \mathbb{E}_{t_{t_{0}}^{\prime}}^{\mathbb{P}^{\prime}} k_{t} d t\right] \leq \varepsilon+\mathbb{E}_{t_{0}}^{\mathbb{P}^{\varepsilon}}\left[\int_{\tau_{0}^{\varepsilon}}^{1} k_{t} d t\right] \leq \varepsilon+2 \varepsilon\left(1-t_{0}\right), \quad \mathbb{P} \text {-a.s. }
$$

By the arbitrariness of $\varepsilon>0$, and the nonnegativity of $k$, this provides (5.6). 


\subsection{Markovian solution of the 2BSDE}

Following the classical terminology in the BSDE literature, we say that the solution of the 2BSDE is Markovian if it can be represented by means of a determinitic function of $\left(t, B_{t}\right)$. In this subsection we construct a deterministic function $u$, by using a probabilistic representation in the spirit of (4.7), and show its connection with 2BSDE (5.4). The connection between $u$ and the PDE (5.5) will be established in the next subsection.

Following [17], we introduce the shifted probability spaces. For $0 \leq t \leq 1$, denote by $\Omega^{t}:=\left\{\omega \in C\left([t, 1], \mathbb{R}^{d}\right): \omega(t)=0\right\}$ the shifted canonical space; $B^{t}$ the shifted canonical process on $\Omega^{t} ; \mathbb{P}_{0}^{t}$ the shifted Wiener measure; $\mathbb{F}^{t}$ the shifted filtration generated by $B^{t}, \overline{\mathcal{P}}_{S}^{t}$ the corresponding collection of martingale measures induced by the strong formulation, and $\hat{a}^{t}$ the universal quadratic variation density of $B^{t}$. In light of Definition 2.6, we define

Definition 5.4 For $t \in[0,1]$, let $\mathcal{P}_{h}^{\kappa, t}$ denote the collection of all those $\mathbb{P} \in \overline{\mathcal{P}}_{S}^{t}$ such that

$$
\begin{aligned}
& \underline{a}_{\mathbb{P}} \leq \hat{a}^{t} \leq \bar{a}_{\mathbb{P}}, \quad d s \times d \mathbb{P} \text {-a.s. on }[t, 1] \times \Omega^{t}, \text { for some } \underline{a}_{\mathbb{P}}, \bar{a}_{\mathbb{P}} \in \mathbb{S}_{d}^{>0}, \\
& \text { and } \left.\mathbb{E}^{\mathbb{P}}\left[\left.\left(\int_{t}^{1} \mid \hat{f}_{s}^{t, 0}\right)\right|^{\kappa} d s\right)^{2 / \kappa}\right]<\infty, \text { where } \hat{f}_{s}^{t, 0}:=f\left(s, 0,0,0, \hat{a}_{s}^{t}\right) .
\end{aligned}
$$

Remark 5.5 By Lemma 6.1 below, $\mathcal{P}_{h}^{\kappa} \neq \varnothing$ implies that $\mathcal{P}_{h}^{\kappa, t} \neq \varnothing$ for all $t \in[0,1]$.

By Assumption 5.1, the polynomial growth of $\rho$, and the first part of (5.7), it is clear that

$\mathbb{E}^{\mathbb{P}}\left[\left(\int_{t}^{1}\left|\hat{f}_{s}^{t, 0}\right|^{\kappa} d s\right)^{2 / \kappa}\right]<\infty$ if and only if $\mathbb{E}^{\mathbb{P}}\left[\left(\int_{t}^{1}\left|f\left(s, B_{s}^{t}, 0,0, \hat{a}_{s}^{t}\right)\right|^{\kappa} d s\right)^{2 / \kappa}\right]<\infty$,

and thus, for $t=0$, we see that $\mathcal{P}_{h}^{\kappa}=\mathcal{P}_{h}^{\kappa, 0}$ as defined in Definition 2.6.

We next define a similar notation to (4.3). For any $(t, x) \in[0,1] \times \mathbb{R}^{d}$, denote

$$
B_{s}^{t, x}:=x+B_{s}^{t} \text { for all } s \in[t, 1] .
$$

Let $\tau$ be and $\mathbb{F}^{t}$-stopping time, $\mathbb{P} \in \mathcal{P}_{h}^{\kappa, t}$, and $\eta$ a $\mathbb{P}$-square integrable $\mathcal{F}_{\tau}^{t}$-measurable r.v. See Remark 2.4. We denote by $\left(\mathcal{Y}^{\mathbb{P}}, \mathcal{Z}^{\mathbb{P}}\right):=\left(\mathcal{Y}^{t, x, \mathbb{P}}(\tau, \eta), \mathcal{Z}^{t, x, \mathbb{P}}(\tau, \eta)\right)$ the solution of the following BSDE:

$$
\mathcal{Y}_{s}^{\mathbb{P}}=\eta-\int_{t}^{\tau} f\left(r, B_{r}^{t, x}, \mathcal{Y}_{r}^{\mathbb{P}}, \mathcal{Z}_{r}^{\mathbb{P}}, \hat{a}_{r}\right) d r-\int_{s}^{\tau} \mathcal{Z}_{r}^{\mathbb{P}} d B_{r}, \quad t \leq s \leq \tau, \quad \mathbb{P} \text {-a.s. }
$$


Similar to (4.3), under our assumptions the above BSDE has a unique solution. We now introduce the value function:

$$
u(t, x):=\sup _{\mathbb{P} \in \mathcal{P}_{h}^{\kappa, t}} \mathcal{Y}_{t}^{t, x, \mathbb{P}}\left(1, g\left(B_{1}^{t, x}\right)\right), \quad \text { for }(t, x) \in[0,1] \times \mathbb{R}^{d} .
$$

By the Blumenthal zero-one law (2.5), it follows that $\mathcal{Y}_{t}^{t, x, \mathbb{P}}\left(1, g\left(B_{1}^{t, x}\right)\right)$ is a constant and thus $u(t, x)$ is deterministic.

Remark 5.6 Notice that, in contrast with the previous sections, we are now implicitly working with the filtration $\mathbb{F}$. However, the subsequent Theorem 5.9 connects $u\left(t, B_{t}\right)$ to the solution of the $2 \mathrm{BSDE}$, implying that $Y$ is $\mathbb{F}$-progressively measurable. See Remark 4.7.

We next state a strengthening of Assumption 4.1 in the present Markov framework.

Assumption 5.7 The function $g$ has polynomial growth, and there exists a continuous positive function $\Lambda(t, x)$ such that, for any $(t, x)$ :

$$
\begin{aligned}
& \sup _{\mathbb{P} \in \mathcal{P}_{h}^{\kappa, t}} \mathbb{E}^{\mathbb{P}}\left[\left|g\left(B_{1}^{t, x}\right)\right|^{\kappa}+\int_{t}^{1}\left|f\left(s, B_{s}^{t, x}, 0,0, \hat{a}_{s}^{t}\right)\right|^{\kappa} d s\right] \leq \Lambda^{\kappa}(t, x), \\
& \sup _{\mathbb{P} \in \mathcal{P}_{h}^{\kappa, t}} \mathbb{E}^{\mathbb{P}}\left[\sup _{t \leq s \leq 1} \Lambda^{2}\left(s, B_{s}^{t, x}\right)\right]<\infty .
\end{aligned}
$$

By the definition of $\Lambda$, it is clear that

$$
|u| \leq \Lambda
$$

Remark 5.8 There are two typical sufficient conditions for the existence of such $\Lambda$ :

(i) $f$ and $g$ are bounded. In this case one can choose $\Lambda$ to be a constant.

(ii) $D_{f}$ is bounded and $\sup _{\mathbb{P} \in \mathcal{P}_{h}^{\kappa, t}} \mathbb{E}^{\mathbb{P}}\left[\int_{t}^{1}\left|\hat{f}_{s}^{t, 0}\right|^{\kappa} d s\right] \leq C$ for all $t$. In this case one can choose $\Lambda$ to be a polynomial of $|x|$.

Theorem 5.9 Let Assumptions 5.1 and 5.7 hold true, and $g$ be uniformly continuous, so that the $2 B S D E$ (5.4) has a unique solution $(Y, Z) \in \mathbb{D}_{H}^{2, \kappa} \times \mathbb{H}_{H}^{2, \kappa}$. Then $Y_{t}=u\left(t, B_{t}\right)$. Moreover, $u$ is uniformly continuous in $x$, uniformly in $t$, and right continuous in $t$.

Proof. The wellposedness of 2BSDE (5.4) follows directly from Theorem 4.6. Notice that $u\left(t, B_{t}\right)=V_{t}$ as defined in [17]. By Remark 4.7, $Y_{t}=V_{t}$, and thus $Y_{t}=u\left(t, B_{t}\right)$.

The uniform continuity of $u$ follows from Lemma 4.6 of [17]; alternatively one can follow the proof of Lemma 4.2 applied to the difference of two solutions. Finally, for any $(t, x)$ and $\delta>0$, the decomposition

$$
|u(t+\delta, x)-u(t, x)|=u(t+\delta, x)-u\left(t+\delta, B_{t+\delta}^{t, x}\right)+Y_{t+\delta}^{t, x}-Y_{t}^{t, x}
$$


implies the right continuity of $u$ in $t$, as a consequence of the uniform continuity of $u$ in $x$, uniformly in $t$, and the right continuity of the process $Y$.

Finally, for later use, we provide an additional regularity result on $u$.

Proposition 5.10 Let Assumptions 5.1 and 5.7 hold true, and $g$ be lower-semi continuous. Then $u$ is lower-semicontinuous in $(t, x)$.

The proof is closely related to the Dynamic Programming Principle, and is postponed to Sect. 6.4.

\subsection{The viscosity solution property}

We shall make use of the classical notations in the theory of viscosity solutions:

$$
\begin{aligned}
& u_{*}(\theta):=\varlimsup_{\theta^{\prime} \rightarrow \theta}^{\lim } u(\theta) \text { and } u^{*}(\theta):=\varlimsup_{\theta^{\prime} \rightarrow \theta} u\left(\theta^{\prime}\right), \quad \text { for } \theta=(t, x) ; \\
& \hat{h}_{*}(\theta):=\varlimsup_{\theta^{\prime} \rightarrow \theta}^{\lim } \hat{h}\left(\theta^{\prime}\right) \text { and } \hat{h}^{*}(\theta):=\varlimsup_{\theta^{\prime} \rightarrow \theta} \hat{h}\left(\theta^{\prime}\right), \quad \text { for } \theta=(t, x, y, z, \gamma) .
\end{aligned}
$$

Theorem 5.11 Let Assumptions 5.1 and 5.7 hold true. Then:

(i) $u$ is a viscosity subsolution of

$$
-\partial_{t} u^{*}-\hat{h}^{*}\left(\cdot, u^{*}, D u^{*}, D^{2} u^{*}\right) \leq 0 \quad \text { on }[0,1) \times \mathbb{R}^{d}
$$

(ii) Assume further that $g$ is lower-semicontinuous and $D_{f}$ is independent of t, then $u$ is a viscosity supersolution of

$$
-\partial_{t} u_{*}-\hat{h}_{*}\left(\cdot, u_{*}, D u_{*}, D^{2} u_{*}\right) \geq 0 \text { on }[0,1) \times \mathbb{R}^{d}
$$

Example 5.12 Let us illustrate the role of $\hat{h}^{*}$ and $\hat{h}_{*}$ in the context of Example 5.2. In this case, one can check immediately that

$$
\hat{h}_{*}=\hat{h} \text { and } \hat{h}^{*}(\gamma)=\frac{1}{2}(\gamma \vee \underline{\Gamma}) \mathbf{1}_{\{\gamma<\bar{\Gamma}\}}+\infty \mathbf{1}_{\{\gamma \geq \bar{\Gamma}\}} .
$$

Then the above viscosity properties are equivalent to

$$
\begin{aligned}
& \min \left\{-\partial_{t} u^{*}-\frac{1}{2}\left(D^{2} u^{*} \vee \underline{\Gamma}\right), \bar{\Gamma}-D^{2} u^{*}\right\} \leq 0, \\
& \min \left\{-\partial_{t} u_{*}-\frac{1}{2}\left(D^{2} u_{*} \vee \underline{\Gamma}\right), \bar{\Gamma}-D^{2} u_{*}\right\} \geq 0,
\end{aligned}
$$

which is exactly the nonlinearity obtained in [3]. 
Remark 5.13 (i) If $u$ is continuous and $D_{\hat{h}}=\mathbb{R}^{d \times d}$, then by Theorem $5.11 u$ is a viscosity solution to PDE (5.5) in the standard sense.

(ii) If the comparison principle for the following relaxed boundary value fully nonlinear PDE (5.14)-(5.15) with boundary condition holds:

$$
\begin{aligned}
& \max \left\{\left(-\partial_{t} v-\hat{h}_{*}\left(\cdot, v, D v, D^{2} v\right)\right)(T, .), v(T, .)-g\right\} \geq 0 \\
& \min \left\{\left(-\partial_{t} v-\hat{h}^{*}\left(\cdot, v, D v, D^{2} v\right)\right)(T, .), v(T, .)-g\right\} \leq 0
\end{aligned}
$$

then $u$ is continuous and is the unique viscosity solution to the above problem. We refer to Crandal et al. [5] for the notion of relaxed boundary problems.

The viscosity property is a consequence of the following dynamic programming principle.

Proposition 5.14 Let $g$ be lower-semicontinuous, $t \in[0,1]$, and $\left\{\tau^{\mathbb{P}}, \mathbb{P} \in \mathcal{P}_{h}^{\kappa, t}\right\}$ be a family of $\mathbb{F}^{t}$-stopping times. Then, under Assumptions 5.1 and 5.7:

$$
u(t, x)=\sup _{\mathbb{P} \in \mathcal{P}_{h}^{\kappa, t}} \mathcal{Y}_{t}^{t, x, \mathbb{P}}\left(\tau^{\mathbb{P}}, u\left(\tau^{\mathbb{P}}, B_{\tau^{\mathbb{P}}}^{t, x}\right)\right) .
$$

The proof of Proposition 5.14 is reported in Sects. 6.2 and 6.4.

Proof of Theorem 5.11 (i) We argue by contradiction, and we aim for a contradiction of the dynamic programming principle. Assume to the contrary that

$$
0=\left(u^{*}-\varphi\right)\left(t_{0}, x_{0}\right)>\left(u^{*}-\varphi\right)(t, x) \text { for all }(t, x) \in\left([0,1] \times \mathbb{R}^{d}\right) \backslash\left\{\left(t_{0}, x_{0}\right)\right\}
$$

for some $\left(t_{0}, x_{0}\right) \in[0,1) \times \mathbb{R}^{d}$ and

$$
\left(-\partial_{t} \varphi-\hat{h}^{*}\left(., \varphi, D \varphi, D^{2} \varphi\right)\right)\left(t_{0}, x_{0}\right)>0
$$

for some smooth function $\varphi$. By (5.12), without loss of generality we may assume $|\varphi| \leq \Lambda$. We note that (5.18) implies that $D^{2} \varphi\left(t_{0}, x_{0}\right) \in D_{\hat{h}}$. Since $\hat{h}^{*}$ is upper-semi continuous and $\varphi$ is smooth, there exists an open ball $O_{r}\left(t_{0}, x_{0}\right)$, centered at $\left(t_{0}, x_{0}\right)$ with radius $r$, such that

$$
-\partial_{t} \varphi-\hat{h}\left(., \varphi, D \varphi, D^{2} \varphi\right) \geq 0 \text {, on } O_{r}\left(t_{0}, x_{0}\right)
$$

Then, we deduce from the definition of $\hat{h}$ that

$$
-\partial_{t} \varphi-\frac{1}{2} \alpha: D^{2} \varphi+f(., \varphi, D \varphi, \alpha) \geq 0 \text { on } O_{r}\left(t_{0}, x_{0}\right) \text { for all } \alpha \in \mathbb{S}_{d}^{>0}(\mathbb{R})
$$


By the strict maximum property (5.17), we notice that

$$
\eta:=-\max _{\partial O_{r}\left(t_{0}, x_{0}\right)}\left(u^{*}-\varphi\right)>0 .
$$

Let $\left(t_{n}, x_{n}\right)$ be a sequence of $O_{r}\left(t_{0}, x_{0}\right)$ such that

$$
\left(t_{n}, x_{n}\right) \longrightarrow\left(t_{0}, x_{0}\right) \text { and } u\left(t_{n}, x_{n}\right) \longrightarrow u^{*}\left(t_{0}, x_{0}\right)
$$

and define the stopping time $\tau_{n}:=\inf \left\{s>t_{n}:\left(s, B_{s}^{t_{n}, x_{n}}\right) \notin O_{r}\left(t_{0}, x_{0}\right)\right\}$. Without loss of generality we may assume $r<1-t_{0}$, then $\tau_{n}<1$ and thus $\left(\tau_{n}, B_{\tau_{n}}^{t_{n}, x_{n}}\right) \in$ $\partial O_{r}\left(t_{0}, x_{0}\right)$. With this construction we have

$$
c_{n}:=(\varphi-u)\left(t_{n}, x_{n}\right) \rightarrow 0 \text { and } u^{*}\left(\tau_{n}, B_{\tau_{n}}^{t_{n}, x_{n}}\right) \leq \varphi\left(\tau_{n}, B_{\tau_{n}}^{t_{n}, x_{n}}\right)-\eta
$$

by the continuity of the coordinate process.

For any $\mathbb{P}^{n} \in \mathcal{P}_{h}^{\kappa, t_{n}}$, we now compute by the comparison result for BSDEs and classical estimates that

$$
\begin{aligned}
& \mathcal{Y}_{t_{n}}^{t_{n}, x_{n}, \mathbb{P}^{n}}\left(\tau_{n}, u^{*}\left(\tau_{n}, B_{\tau_{n}}^{t_{n}, x_{n}}\right)\right)-u\left(t_{n}, x_{n}\right) \\
& \quad \leq \mathcal{Y}_{t_{n}}^{t_{n}, x_{n}, \mathbb{P}^{n}}\left(\tau_{n}, \varphi\left(\tau_{n}, B_{\tau_{n}}^{t_{n}, x_{n}}\right)-\eta\right)-\varphi\left(t_{n}, x_{n}\right)+c_{n} \\
& \quad \leq \mathcal{Y}_{t_{n}}^{t_{n}, x_{n}, \mathbb{P}^{n}}\left(\tau_{n}, \varphi\left(\tau_{n}, B_{\tau_{n}}^{t_{n}, x_{n}}\right)\right)-\varphi\left(t_{n}, x_{n}\right)+c_{n}-\eta^{\prime}
\end{aligned}
$$

for some positive constant $\eta^{\prime}$ independent of $n$. Set

$$
\begin{aligned}
& \left(Y^{n}, Z^{n}\right):=\left(\mathcal{Y}^{t_{n}, x_{n}, \mathbb{P}^{n}}, \mathcal{Z}^{t_{n}, x_{n}, \mathbb{P}^{n}}\right)\left(\tau_{n}, \varphi\left(\tau_{n}, B_{\tau_{n}}^{t_{n}, x_{n}}\right)\right), \\
& \delta Y_{s}^{n}:=Y_{s}^{n}-\varphi\left(s, B_{s}^{t_{n}, x_{n}}\right), \text { and } \delta Z_{s}^{n}:=Z_{s}^{n}-D \varphi\left(s, B_{s}^{t_{n}, x_{n}}\right) .
\end{aligned}
$$

It follows from Itô's formula together with the Lipschitz properties of $f$ that, $\mathbb{P}^{n}$-a.s.

$$
\begin{aligned}
d\left(\delta Y_{s}^{n}\right) & =\left(-\partial_{t} \varphi-\frac{1}{2} \hat{a}_{s}: D^{2} \varphi+f\left(., Y_{s}^{n}, Z_{s}^{n}, \hat{a}_{s}\right)\right)\left(s, B_{s}^{t_{n}, x_{n}}\right) d s+\delta Z_{s}^{n} d B_{s} \\
& =\left(\phi_{s}^{n}+\lambda_{s} \delta Y_{s}^{n}+\delta Z_{s}^{n} \bar{\alpha}^{1 / 2} \beta_{s}\right) d s+\delta Z_{s}^{n} d B_{s}
\end{aligned}
$$

where $\lambda$ and $\beta$ are bounded progressively measurable processes, and

$$
\phi_{s}^{n}:=\left(-\partial_{t} \varphi-\frac{1}{2} \hat{a}_{s}: D^{2} \varphi+f\left(., \varphi, D \varphi, \hat{a}_{s}\right)\right)\left(s, B_{s}^{t_{n}, x_{n}}\right) \geq 0 \text { for } s \in\left[t_{n}, \tau_{n}\right]
$$

by (5.25) and the definition of $\tau_{n}$. Let $M$ be defined by (4.10), but starting from $t_{n}$ and under $\mathbb{P}^{n}$. Then

$$
\mathcal{Y}_{t_{n}}^{t_{n}, x_{n}, \mathbb{P}^{n}}\left(\tau_{n}, \varphi\left(\tau_{n}, B_{\tau_{n}}^{t_{n}, x_{n}}\right)\right)-\varphi\left(t_{n}, x_{n}\right)=\delta Y_{t_{n}}^{n} \leq \mathbb{E}^{\mathbb{P}^{n}}\left[M_{\tau_{n}} \delta Y_{\tau_{n}}^{n}\right]=0
$$


Plugging this in (5.22), we get

$$
\mathcal{Y}_{t_{n}}^{t_{n}, x_{n}, \mathbb{P}^{n}}\left(\tau_{n}, u^{*}\left(\tau_{n}, B_{\tau_{n}}^{t_{n}, x_{n}}\right)\right)-u\left(t_{n}, x_{n}\right) \leq c_{n}-\eta^{\prime}
$$

Note that $\mathbb{P}^{n} \in \mathcal{P}_{h}^{\kappa, t_{n}}$ is arbitrary and $c_{n}$ does not depend on $\mathbb{P}^{n}$. Then

$$
\sup _{\mathbb{P} \in \mathcal{P}_{h}^{\kappa, t_{n}}} \mathcal{Y}_{t_{n}}^{t_{n}, x_{n}, \mathbb{P}}\left(\tau_{n}, u^{*}\left(\tau_{n}, B_{\tau_{n}}^{t_{n}, x_{n}}\right)\right)-u\left(t_{n}, x_{n}\right) \leq c_{n}-\eta^{\prime}<0,
$$

for large $n$. This is in contradiction with the dynamic programming principle of Proposition 5.14 (or, more precisely, Lemma 6.2 below to avoid the condition that $g$ is lower-semicontinuous).

(ii) We again argue by contradiction, aiming for a contradiction of the dynamic programming principle of Proposition 5.14. Assume to the contrary that

$$
0=\left(u_{*}-\varphi\right)\left(t_{0}, x_{0}\right)<\left(u_{*}-\varphi\right)(t, x) \text { for all }(t, x) \in\left([0,1] \times \mathbb{R}^{d}\right) \backslash\left\{\left(t_{0}, x_{0}\right)\right\}
$$

for some $\left(t_{0}, x_{0}\right) \in[0,1) \times \mathbb{R}^{d}$ and

$$
\left(-\partial_{t} \varphi-\hat{h}_{*}\left(., \varphi, D \varphi, D^{2} \varphi\right)\right)\left(t_{0}, x_{0}\right)<0,
$$

for some smooth function $\varphi$. By (5.12), without loss of generality we may assume again that $|\varphi| \leq \Lambda$. Note that $\hat{h}_{*} \leq \hat{h}$. Then

$$
\left(-\partial_{t} \varphi-\hat{h}\left(., \varphi, D \varphi, D^{2} \varphi\right)\right)\left(t_{0}, x_{0}\right)<0
$$

If $D^{2} \varphi\left(t_{0}, x_{0}\right) \in D_{\hat{h}}$, then it follows from the definition of $\hat{h}$ that

$$
\left(-\partial_{t} \varphi-\frac{1}{2} \bar{\alpha}: D^{2} \varphi+f(., \varphi, D \varphi, \bar{\alpha})\right)\left(t_{0}, x_{0}\right)<0
$$

for some $\bar{\alpha} \in \mathbb{S}_{d}^{>0}$. In particular, this implies that $\bar{\alpha} \in D_{f}$. If $D^{2} \varphi\left(t_{0}, x_{0}\right) \notin D_{\hat{h}}$, since $\partial_{t} \varphi\left(t_{0}, x_{0}\right)$ is finite, we still have $\bar{\alpha} \in D_{f}$ so that (5.24) holds. Now by the smoothness of $\varphi$ and (5.3), and recalling that $D_{f}$ is independent of $t$, there exists an open ball $O_{r}\left(t_{0}, x_{0}\right)$ with $0<r<1-t_{0}$ such that

$$
-\partial_{t} \varphi-\frac{1}{2} \bar{\alpha}: D^{2} \varphi+f(., \varphi, D \varphi, \bar{\alpha}) \leq 0 \text { on } O_{r}\left(t_{0}, x_{0}\right)
$$

By the strict minimum property (5.23), we notice that

$$
\eta:=\min _{\partial B_{r}\left(t_{0}, x_{0}\right)}\left(u_{*}-\varphi\right)>0 .
$$


As in (i), we consider a sequence $\left(t_{n}, x_{n}\right)$ of $O_{r}\left(t_{0}, x_{0}\right)$ such that

$$
\left(t_{n}, x_{n}\right) \longrightarrow\left(t_{0}, x_{0}\right) \text { and } u\left(t_{n}, x_{n}\right) \longrightarrow u_{*}\left(t_{0}, x_{0}\right)
$$

and we define the stopping time $\tau_{n}:=\inf \left\{s>t_{n}:\left(s, B_{s}^{t_{n}, x_{n}}\right) \notin O_{r}\left(t_{0}, x_{0}\right)\right\}$, so that

$$
c_{n}:=(u-\varphi)\left(t_{n}, x_{n}\right) \rightarrow 0 \text { and } u_{*}\left(\tau_{n}, B_{\tau_{n}}^{t_{n}, x_{n}}\right) \geq \varphi\left(\tau_{n}, B_{\tau_{n}}^{t_{n}, x_{n}}\right)+\eta
$$

For each $n$, let $\overline{\mathbb{P}}^{n}:=\mathbb{P}^{\bar{\alpha}} \in \overline{\mathcal{P}}_{S}^{t_{n}}$ be the local martingale measure induced by the constant diffusion $\bar{\alpha}$. By (5.3), one can easily see that $\overline{\mathbb{P}}^{n} \in \mathcal{P}_{H}^{\kappa, t_{n}}$. We then follow exactly the same line of argument as in (i) to see that

$$
u\left(t_{n}, x_{n}\right)-\mathcal{Y}_{t_{n}}^{t_{n}, x_{n}, \overline{\mathbb{P}}^{n}}\left(\tau_{n}, u_{*}\left(\tau_{n}, B_{\tau_{n}}^{t_{n}, x_{n}}\right)\right) \leq c_{n}-\eta^{\prime}, \quad \overline{\mathbb{P}} \text {-a.s. }
$$

where $\eta^{\prime}$ is a positive constant independent of $n$. For large $n$, we have $c_{n}-\eta^{\prime}<0$, and this is in contradiction with the dynamic programming principle.

\section{The dynamic programming principle}

In this section we prove Propositions 5.14 and 5.10.

\subsection{Regular conditional probability distributions}

The key tool to prove the dynamic programming principle is the regular conditional probability distributions (r.c.p.d.), introduced by Stroock-Varadhan [18]. We adopt the notations of our accompanying paper [17]. For $0 \leq t \leq s \leq 1, \omega \in \Omega^{t}, \tilde{\omega} \in \Omega^{s}$, and $\mathcal{F}_{1}^{t}$-measurable random variable $\xi$, define:

$$
\begin{aligned}
\xi^{s, \omega}(\tilde{\omega}) & :=\xi\left(\omega \otimes_{s} \tilde{\omega}\right) \text { where }\left(\omega \otimes_{s} \tilde{\omega}\right)(r) \\
& :=\omega_{r} \mathbf{1}_{[t, s)}(r)+\left(\omega_{s}+\tilde{\omega}_{r}\right) \mathbf{1}_{[s, 1]}(r), \quad r \in[t, 1] .
\end{aligned}
$$

In particular, for any $\mathbb{F}^{t}$-stopping time $\tau$, one can choose $s=\tau(\omega)$ and simplify the notation: $\omega \otimes_{\tau} \tilde{\omega}:=\omega \otimes_{\tau(\omega)} \tilde{\omega}$. Clearly $\omega \otimes_{\tau} \tilde{\omega} \in \Omega^{t}$ and, for each $\omega \in \Omega^{t}, \xi^{\tau, \omega}:=$ $\xi^{\tau(\omega), \omega}$ is $\mathcal{F}_{1}^{\tau(\omega)}$-measurable. For each probability measure $\mathbb{P}$ on $\left(\Omega^{t}, \mathcal{F}_{1}^{t}\right)$, by StroockVaradhan [18] there exist r.c.p.d. $\mathbb{P}^{\tau, \omega}$ for all $\omega \in \Omega^{t}$ such that $\mathbb{P}^{\tau, \omega}$ is a probability measure on $\left(\Omega^{\tau(\omega)}, \mathcal{F}_{1}^{\tau(\omega)}\right)$, and for all $\mathcal{F}_{1}^{t}$-measurable $\mathbb{P}$-integrable random variable $\xi$ :

$$
\mathbb{E}^{\mathbb{P}}\left[\xi \mid \mathcal{F}_{\tau}^{t}\right](\omega)=\mathbb{E}^{\mathbb{P}^{\tau, \omega}}\left[\xi^{\tau, \omega}\right], \quad \text { for } \mathbb{P} \text {-a.e. } \omega \in \Omega^{t}
$$

In particular, this implies that the mapping $\omega \mapsto \mathbb{E}^{\mathbb{P}^{\tau, \omega}}\left[\xi^{\tau, \omega}\right]$ is $\mathcal{F}_{\tau}^{t}$-measurable. Moreover, following the arguments in Lemmas 4.1 and 4.3 of [17], one can easily show that: 
Lemma 6.1 Let $t \in[0,1], \tau$ an $\mathbb{F}^{t}$-stopping time, and $\mathbb{P} \in \mathcal{P}_{h}^{\kappa, t}$. Then:

$$
\begin{aligned}
& \text { for } \mathbb{P} \text {-a.e. } \omega \in \Omega^{t}: \mathbb{P}^{\tau, \omega} \in \mathcal{P}_{h}^{\kappa, \tau(\omega)} \text { and }\left(\hat{a}^{t}\right)_{r}^{\tau, \omega}=\hat{a}_{r}^{\tau(\omega)}, \\
& \qquad d r \times d \mathbb{P}^{\tau, \omega} \text { on }[\tau(\omega), 1] \times \Omega^{\tau(\omega)} \text {. }
\end{aligned}
$$

\subsection{A weak partial dynamic programming principle}

In this section, we prove the following result adapted from [2].

Lemma 6.2 Under Assumptions 5.1 and 5.7, for any $(t, x)$ and arbitrary $\mathbb{F}^{t}$-stopping times $\left\{\tau^{\mathbb{P}}, \mathbb{P} \in \mathcal{P}_{h}^{\kappa, t}\right\}$ :

$$
u(t, x) \leq \sup _{\mathbb{P} \in \mathcal{P}_{h}^{\kappa, t}} \mathcal{Y}_{t}^{t, x, \mathbb{P}}\left(\tau^{\mathbb{P}}, u^{*}\left(\tau^{\mathbb{P}}, B_{\tau^{\mathbb{P}}}^{t, x}\right)\right) .
$$

Proof. We shall prove the slightly stronger result:

$$
\begin{aligned}
& \mathcal{Y}_{t}^{t, x, \mathbb{P}}\left(1, g\left(B_{1}^{t, x}\right)\right) \leq \mathcal{Y}_{t}^{t, x, \mathbb{P}}\left(\tau^{\mathbb{P}}, \varphi\left(\tau^{\mathbb{P}}, B_{\tau^{\mathbb{P}}}^{t, x}\right)\right) \\
& \text { for any } \mathbb{P} \in \mathcal{P}_{h}^{\kappa, t} \text { and any Lebesgue measurable function } \varphi \geq u .
\end{aligned}
$$

Fix $\mathbb{P}$ and $\varphi$. For notation simplicity, we omit the dependence of $\tau^{\mathbb{P}}$ on $\mathbb{P}$. We first note that, by 5.12, without loss of generality we may assume $|\varphi| \leq \Lambda$. Then Assumption 5.7 implies that $\mathcal{Y}_{t}^{t, x, \mathbb{P}}\left(\tau, \varphi\left(\tau, B_{\tau}^{t, x}\right)\right)$ is well defined. By (6.2), one can easily show that

$$
\mathcal{Y}_{t}^{t, x, \mathbb{P}}\left(1, g\left(B_{1}^{t, x}\right)\right)=\mathcal{Y}_{t}^{t, x, \mathbb{P}}\left(\tau, \mathcal{Y}_{\tau}^{\tau(\omega), B_{\tau}^{t, x}(\omega), \mathbb{P}^{\tau, \omega}}\left(1, g\left(B_{1}^{\tau(\omega), B_{\tau}^{t, x}(\omega)}\right)\right)\right)
$$

By Lemma 6.1, $\mathbb{P}^{\tau, \omega} \in \mathcal{P}_{h}^{\kappa, \tau(\omega)}, \mathbb{P}$-a.e. $\omega \in \Omega^{t}$. Then

$$
\begin{aligned}
& \mathcal{Y}_{\tau}^{\tau(\omega), B_{\tau}^{t, x}(\omega), \mathbb{P}^{\tau, \omega}}\left(1, g\left(B_{1}^{\tau(\omega), B_{\tau}^{t, x}(\omega)}\right)\right) \\
& \quad \leq u\left(\tau(\omega), B_{\tau}^{t, x}(\omega)\right) \leq \varphi\left(\tau(\omega), B_{\tau}^{t, x}(\omega)\right), \quad \text { P-a.e. } \omega \in \Omega^{t} .
\end{aligned}
$$

It follows from the comparison result for BSDEs that

$$
\mathcal{Y}_{t}^{t, x, \mathbb{P}}\left(1, g\left(B_{1}^{t, x}\right)\right) \leq \mathcal{Y}_{t}^{t, x, \mathbb{P}}\left(\tau, \varphi\left(\tau, B_{\tau}^{t, x}\right)\right)
$$

This implies (6.3), and by the arbitrariness of $\mathbb{P}$, Lemma 6.2 is proved.

\subsection{Concatenation of probability measures}

In preparation to the proof of Proposition 5.14, we introduce the concatenation of probability measures. For any $0 \leq t_{0} \leq t \leq 1$ and $\omega \in \Omega^{t_{0}}$, denote $\omega^{t} \in \Omega^{t}$ 
by $\omega_{s}^{t}:=\omega_{s}-\omega_{t}, s \in[t, 1]$. For any $\mathbb{P}_{1}=\mathbb{P}^{\alpha^{1}} \in \mathcal{P}_{h}^{\kappa, t_{0}}, \mathbb{P}_{2}=\mathbb{P}^{\alpha^{2}} \in \mathcal{P}_{h}^{\kappa, t}$, let $\mathbb{P}:=\mathbb{P}_{1} \otimes_{t} \mathbb{P}_{2}$ denote the probability measure $\mathbb{P}^{\alpha}$, where

$$
\alpha_{s}(\omega):=\alpha_{s}^{1}(\omega) \mathbf{1}_{\left[t_{0}, t\right]}(s)+\alpha_{s}^{2}\left(\omega^{t}\right) \mathbf{1}_{[t, 1]}(s), \quad \omega \in \Omega^{t_{0}}
$$

Lemma 6.3 Let $\mathbb{P}:=\mathbb{P}_{1} \otimes_{t} \mathbb{P}_{2}$ be as defined above. Then, under Assumption 5.1,

$$
\mathbb{P} \in \mathcal{P}_{h}^{\kappa, t_{0}}, \mathbb{P}=\mathbb{P}_{1} \text { on } \mathcal{F}_{t}^{t_{0}}, \quad \text { and } \quad \mathbb{P}^{t, \omega}=\mathbb{P}_{2} \text { for } \mathbb{P}_{1} \text {-a.e. } \omega \in \Omega^{t_{0}}
$$

Proof. First by (5.7), we have $\underline{a}_{\mathbb{P}_{i}} \leq \alpha^{i} \leq \bar{a}_{\mathbb{P}_{i}}, i=1,2$. Then $\underline{a}_{\mathbb{P}_{1}} \wedge \underline{a}_{\mathbb{P}_{2}} \leq$ $\alpha \leq \bar{a}_{\mathbb{P}_{1}} \vee \bar{a}_{\mathbb{P}_{1}}$. In particular, this implies that $\int_{t_{0}}^{1}\left|\alpha_{s}\right| d s<\infty$. Then $\mathbb{P} \in \overline{\mathcal{P}}_{S}^{t_{0}}$ and $\underline{a}_{\mathbb{P}_{1}} \wedge \underline{a}_{\mathbb{P}_{2}} \leq \hat{a} \leq \bar{a}_{\mathbb{P}_{1}} \vee \bar{a}_{\mathbb{P}_{2}}, \mathbb{P}$-a.s. The two last claims in (6.4) are obvious, and imply that:

$$
\begin{aligned}
& \mathbb{E}^{\mathbb{P}}\left[\left(\int_{t_{0}}^{1}\left|\hat{f}_{s}^{t_{0}, 0}\right|^{\kappa} d s\right)^{2 / \kappa}\right] \leq C_{\kappa} \mathbb{E}^{\mathbb{P}}\left[\left(\int_{t_{0}}^{t}\left|\hat{f}_{s}^{t_{0}, 0}\right|^{\kappa} d s\right)^{2 / \kappa}+\left(\int_{t}^{1}\left|\hat{f}_{s}^{t_{0}, 0}\right|^{\kappa} d s\right)^{2 / \kappa}\right] \\
& =C_{\kappa}\left(\mathbb{E}^{\mathbb{P}_{1}}\left[\left(\int_{t_{0}}^{t}\left|\hat{f}_{s}^{t_{0}, 0}\right|^{\kappa} d s\right)^{2 / \kappa}\right]+\mathbb{E}^{\mathbb{P}_{1}}\left[\mathbb{E}^{\mathbb{P}_{2}}\left[\left(\int_{t}^{1}\left|\hat{f}_{s}^{t, 0}\right|^{\kappa} d s\right)^{2 / \kappa}\right]\right]\right) \\
& =C_{\kappa}\left(\mathbb{E}^{\mathbb{P}_{1}}\left[\left(\int_{t_{0}}^{t}\left|\hat{f}_{s}^{t_{0}, 0}\right|^{\kappa} d s\right)^{2 / \kappa}\right]+\mathbb{E}^{\mathbb{P}_{2}}\left[\left(\int_{t}^{1}\left|\hat{f}_{s}^{t, 0}\right|^{\kappa} d s\right)^{2 / \kappa}\right]\right)<\infty .
\end{aligned}
$$

This implies that $\mathbb{P} \in \mathcal{P}_{h}^{\kappa, t_{0}}$.

\subsection{Dynamic programming and regularity}

We first prove the dynamic programming principle of Proposition 5.14 for stopping times taking countably many values. From this, we will deduce the lower-semi continuity of $u$ stated in Proposition 5.10, which in turn provides Proposition 5.14 by passing to limits.

Lemma 6.4 Proposition 5.14 holds true under the additional condition that each $\tau^{\mathbb{P}}$ takes countable many values.

Proof. (i) We first observe that the lower semicontinuity of $g$ implies that

$$
x \longmapsto \mathcal{Y}_{t}^{t, x, \mathbb{P}}\left(1, g\left(B_{1}^{t, x}\right)\right) \text { is lower-semicontinuous for all } \mathbb{P} \in \mathcal{P}_{h}^{\kappa, t} \text {. }
$$


This is a direct consequence of the stability and comparison principle of BSDEs. Then, for all fixed $(t, x)$, and all sequence $\left(x_{n}\right)_{n \geq 1}$ converging to $x$, it follows that:

$$
\begin{aligned}
u(t, x) & =\sup _{\mathbb{P} \in \mathcal{P}_{h}^{\kappa, t}} \mathcal{Y}_{t}^{t, x, \mathbb{P}}\left(1, g\left(X_{1}^{t, x}\right)\right) \\
& \leq \sup _{\mathbb{P} \in \mathcal{P}_{h}^{\kappa, t}} \underline{\lim }_{n \rightarrow \infty} \mathcal{Y}_{t}^{t, x_{n}, \mathbb{P}}\left(1, g\left(X_{1}^{t, x_{n}}\right)\right) \leq \varliminf_{n \rightarrow \infty}^{\lim } u\left(t, x_{n}\right) .
\end{aligned}
$$

Hence $u(t,$.$) is lower-semicontinuous, and therefore measurable.$

(ii) We now fix $\left(t_{0}, x_{0}\right)$ and prove the result at this point. Let $\tau$ be an $\mathbb{F}^{t_{0}}$-stopping time with values in $\left\{t_{k}, k \geq 1\right\} \subset\left[t_{0}, 1\right]$. Since $u\left(t_{k},.\right)$ is measurable, we deduce that $u\left(\tau, B_{\tau}^{t_{0}, x_{0}}\right)=\sum_{k \geq 1} u\left(t_{k}, B_{t_{k}}^{t_{0}, x_{0}}\right) \mathbf{1}_{\left\{\tau=t_{k}\right\}}$ is $\mathcal{F}_{\tau}$-measurable. Then, it follows from (6.3) that

$$
u\left(t_{0}, x_{0}\right) \leq \sup _{\mathbb{P} \in \mathcal{P}_{h}^{\kappa, t_{0}}} \mathcal{Y}_{t_{0}}^{t_{0}, x_{0}, \mathbb{P}}\left(\tau^{\mathbb{P}}, u\left(\tau^{\mathbb{P}}, B_{\tau^{\mathbb{P}}}^{t_{0}, x_{0}}\right)\right)
$$

(iii) To complete the proof, we fix $\mathbb{P} \in \mathcal{P}_{h}^{\kappa, t_{0}}$, denote $\tau:=\tau^{\mathbb{P}}$, and proceed in four steps to show that

$$
\mathcal{Y}_{t_{0}}^{t_{0}, x_{0}, \mathbb{P}}\left(\tau, u\left(\tau, B_{\tau}^{t_{0}, x_{0}}\right)\right) \leq u\left(t_{0}, x_{0}\right)
$$

Step 1. We first fix $t \in\left(t_{0}, 1\right]$, and show that,

$$
\mathcal{Y}_{t_{0}}^{t_{0}, x_{0}, \mathbb{P}}\left(t, \varphi\left(B_{t}^{t_{0}, x_{0}}\right)\right) \leq u\left(t_{0}, x_{0}\right)
$$

for any continuous function $\varphi: \mathbb{R}^{d} \longrightarrow \mathbb{R}$ such that $-\Lambda(t, \cdot) \leq \varphi(\cdot) \leq u(t, \cdot)$. Indeed, for any $\mathbb{P}^{t} \in \mathcal{P}_{h}^{\kappa, t}$, by the lower-semicontinuity property (6.5), we may argue exactly as in Step 2 of the proof of Theorem 3.1 in [2] to deduce that, for every $\varepsilon>0$, there exist sequences $\left(x_{i}, r_{i}\right)_{i \geq 1} \subset \mathbb{R}^{d} \times(0,1]$ and $\mathbb{P}_{i} \in \mathcal{P}_{h}^{\kappa, t}, i \geq 1$ such that

$$
\begin{aligned}
& \mathcal{Y}_{t}^{t, \cdot, \mathbb{P}_{i}}\left(1, g\left(B_{1}^{t, \cdot}\right)\right) \geq \varphi(t, \cdot)-\varepsilon \text { on } Q_{i}:=\left\{x^{\prime} \in \mathbb{R}^{d}:\left|x^{\prime}-x_{i}\right|<r_{i}\right\}, \text { and } \\
& \cup_{i \geq 1} Q_{i}=\mathbb{R}^{d}
\end{aligned}
$$

This provides a disjoint partition $\left(A_{i}\right)_{i \geq 1}$ of $\mathbb{R}^{d}$ defined by $A_{i}:=Q_{i} \backslash \cup_{j<i} Q_{j}$. Set

$$
E_{i}:=\left\{B_{t}^{t_{0}, x_{0}} \in A_{i}\right\}, \quad i \geq 1, \text { and } \bar{E}_{n}:=\cup_{i>n} E_{i}, \quad n \geq 1 .
$$

Then $\left\{E_{i}, 1 \leq i \leq n\right\}$ and $\bar{E}^{n}$ form a partition of $\Omega$ and $\lim _{n \rightarrow \infty} \mathbb{P}\left(\bar{E}_{n}\right)=0$. Define

$$
\overline{\mathbb{P}}^{n}(E):=\sum_{i=1}^{n}\left(\mathbb{P} \otimes_{t} \mathbb{P}_{i}\right)\left(E \cap E_{i}\right)+\mathbb{P}\left(E \cap \bar{E}_{n}\right) \text { for all } E \in \mathcal{F}_{1}^{t_{0}}
$$


Combining the arguments for (4.17) and Lemma 6.3, one can easily show that

$$
\overline{\mathbb{P}}^{n} \in \mathcal{P}_{h}^{\kappa, t_{0}}(t, \mathbb{P}) \text { and }\left(\overline{\mathbb{P}}^{n}\right)^{t, \omega}=\mathbb{P}_{i}, \quad \mathbb{P} \text {-a.e. } \omega \in E_{i}, \quad 1 \leq i \leq n
$$

This implies that, for $1 \leq i \leq n$ and $\mathbb{P}$-a.e. $\omega \in E_{i}$,

$\mathcal{Y}_{t}^{t_{0}, x_{0}, \overline{\mathbb{P}}^{n}}\left(1, g\left(B_{1}^{t_{0}, x_{0}}\right)\right)(\omega)=\mathcal{Y}_{t}^{t, B_{t}^{t_{0}, x_{0}}(\omega), \mathbb{P}_{i}}\left(1, g\left(B_{1}^{t, B^{t_{0}, x_{0}}(\omega)_{t}}\right)\right) \geq \varphi\left(B_{t}^{t_{0}, x_{0}}(\omega)\right)-\varepsilon$

and, by the comparison result for BSDEs:

$$
\begin{aligned}
u\left(t_{0}, x_{0}\right) & \geq \mathcal{Y}_{t_{0}}^{t_{0}, x_{0}, \overline{\mathbb{P}}^{n}}\left(1, g\left(B_{1}^{t_{0}, x_{0}}\right)\right)=\mathcal{Y}_{t_{0}}^{t_{0}, x_{0}, \mathbb{P}}\left(t, \mathcal{Y}_{t}^{t_{0}, x_{0}, \overline{\mathbb{P}}^{n}}\left(1, g\left(B_{1}^{t_{0}, x_{0}}\right)\right)\right) \\
& \geq \mathcal{Y}_{t_{0}}^{t_{0}, x_{0}, \mathbb{P}}\left(t,\left(\varphi\left(B_{t}^{t_{0}, x_{0}}\right)-\varepsilon\right) \mathbf{1}_{\left(\bar{E}_{n}\right)^{c}}+\mathcal{Y}_{t}^{t_{0}, x_{0}, \overline{\mathbb{P}}^{n}}\left(1, g\left(B_{1}^{t_{0}, x_{0}}\right)\right) \mathbf{1}_{\bar{E}_{n}}\right) .
\end{aligned}
$$

By the stability of BSDEs and the arbitrariness of $\varepsilon>0$, this proves (6.7).

Step 2. Since $u(t, \cdot)$ is lower semi-continuous, there exist continuous functions $\left\{\varphi_{n}, n \geq\right.$ $1\}$ such that $\varphi_{n} \uparrow u(t, \cdot)$. Without loss of generality we may assume $\varphi_{n} \geq-\Lambda$. Since (6.7) holds for each $\varphi_{n}$, we obtain (6.6) for $\tau=t$ by monotone convergence.

Step 3. Assume $\tau$ takes finitely many values $t_{0}<t_{1}<\cdots<t_{n} \leq 1$. Note that, $\mathbb{P}$-a.s.

$$
\begin{aligned}
& \mathcal{Y}_{\tau \wedge t_{n-1}}^{t_{0}, x_{0}, \mathbb{P}}\left(\tau, u\left(\tau, B_{\tau}^{t_{0}, x_{0}}\right)\right) \\
& =\mathcal{Y}_{\tau}^{t_{0}, x_{0}, \mathbb{P}}\left(\tau, u\left(\tau, B_{\tau}^{t_{0}, x_{0}}\right)\right) \mathbf{1}_{\left\{\tau \leq t_{n-1}\right\}}+\mathcal{Y}_{t_{n-1}}^{t_{0}, x_{0}, \mathbb{P}}\left(\tau, u\left(\tau, B_{\tau}^{t_{0}, x_{0}}\right)\right) \mathbf{1}_{\left\{\tau>t_{n-1}\right\}} \\
& =u\left(\tau, B_{\tau}^{t_{0}, x_{0}}\right) \mathbf{1}_{\left\{\tau \leq t_{n-1}\right\}}+\mathcal{Y}_{t_{n-1}}^{t_{n-1}, B_{t_{n-1}}^{t_{0}, x_{0}}(\omega),(\mathbb{P})^{t_{n-1}, \omega}}\left(t_{n}, u\left(t_{n}, B_{t_{n}}^{t_{n-1}, B_{t_{n-1}}^{t_{0}, x_{0}}(\omega)}\right)\right) \mathbf{1}_{\left\{\tau>t_{n-1}\right\}}
\end{aligned}
$$

By Lemma $6.1,(\mathbb{P})^{t_{n-1}, \omega} \in \mathcal{P}_{h}^{\kappa, t_{n-1}}, \mathbb{P}$-a.s. Then by Step 2 we have

$$
\begin{aligned}
\mathcal{Y}_{\tau \wedge t_{n-1}}^{t_{0}, x_{0}, \mathbb{P}}\left(\tau, u\left(\tau, B_{\tau}^{t_{0}, x_{0}}\right)\right) & \leq u\left(\tau, B_{\tau}^{t_{0}, x_{0}}\right) \mathbf{1}_{\left\{\tau \leq t_{n-1}\right\}}+u\left(t_{n-1}, B_{t_{n-1}}^{t_{0}, x_{0}}\right) \mathbf{1}_{\left\{\tau>t_{n-1}\right\}} \\
& =u\left(\tau \wedge t_{n-1}, B_{\tau \wedge t_{n-1}}^{t_{0}, x_{0}}\right) .
\end{aligned}
$$

Then, by the comparison principle of BSDE,

$$
\begin{aligned}
\mathcal{Y}_{t_{0}}^{t_{0}, x_{0}, \mathbb{P}}\left(\tau, u\left(\tau, B_{\tau}^{t_{0}, x_{0}}\right)\right) & =\mathcal{Y}_{t_{0}}^{t_{0}, x_{0}, \mathbb{P}}\left(\tau \wedge t_{n-1}, \mathcal{Y}_{\tau \wedge t_{n-1}}^{t_{0}, x_{0}, \mathbb{P}}\left(\tau, u\left(\tau, B_{\tau}^{t_{0}, x_{0}}\right)\right)\right) \\
& \leq \mathcal{Y}_{t_{0}}^{t_{0}, x_{0}, \mathbb{P}}\left(\tau \wedge t_{n-1}, u\left(\tau \wedge t_{n-1}, B_{\tau \wedge t_{n-1}}^{t_{0}, x_{0}}\right)\right) .
\end{aligned}
$$

Continuing this backward induction provides (6.6).

Step 4. Now assume $\tau$ takes countable many values $\left\{t_{k}, k \geq 1\right\}$. Denote $\tau_{n}:=$ $\sum_{k=1}^{n} t_{k} \mathbf{1}_{\left\{\tau=t_{k}\right\}}+\mathbf{1}_{\left\{\tau \neq t_{k}, 1 \leq k \leq n\right\}}$. Clearly $\tau_{n}$ is still an $\mathbb{F}^{t_{0}}$-stopping time. By Step 3 ,

$$
\mathcal{Y}_{t_{0}}^{t_{0}, x_{0}, \mathbb{P}}\left(\tau_{n}, u\left(\tau_{n}, B_{\tau_{n}}^{t_{0}, x_{0}}\right)\right) \leq u\left(t_{0}, x_{0}\right)
$$


For each $\omega \in \Omega^{t_{0}}$, we have $\tau_{n}(\omega)=\tau(\omega)$, for sufficiently large $n$. Then $u\left(\tau_{n}(\omega)\right.$, $\left.B_{\tau_{n}}^{t_{0}, x_{0}}(\omega)\right)=u\left(\tau(\omega), B_{\tau}^{t_{0}, x_{0}}(\omega)\right)$, and (6.6) follows from the stability of BSDEs.

As a consequence of Lemma 6.4, we can now prove that $u$ is lower-semicontinuous.

Proof of Proposition 5.10 Recall the $\mathcal{Y}^{\mathbb{P}}(\tau, \xi)$ defined in (4.3), and define

$$
J(t, x, \mathbb{P}):=\mathbb{E}^{\mathbb{P}}\left[\mathcal{Y}_{t}^{\mathbb{P}}\left(1, g\left(x+B_{1}-B_{t}\right)\right)\right] \text { for all } t, x, \text { and } \mathbb{P} \in \mathcal{P}_{h}^{\kappa}
$$

(i) We first prove that

$$
u(t, x)=\sup _{\mathbb{P} \in \mathcal{P}_{h}^{\kappa}} J(t, x, \mathbb{P})
$$

To see this, we first observe that, for any $\mathbb{P} \in \mathcal{P}_{h}^{\kappa}$, it follows from Lemma 6.1 that

$\mathcal{Y}_{t}^{\mathbb{P}}\left(1, g\left(x+B_{1}-B_{t}\right)\right)(\omega)=\mathcal{Y}_{t}^{t, x, \mathbb{P}^{t, \omega}}\left(1, g\left(B_{1}^{t, x}\right)\right) \leq u(t, x)$ for $\mathbb{P}$-a.e. $\omega \in \Omega$.

Then $J(t, x, \mathbb{P}) \leq u(t, x)$ for any $\mathbb{P} \in \mathcal{P}_{h}^{\kappa}$.

On the other hand, for any $\mathbb{P}_{2} \in \mathcal{P}_{h}^{\kappa, t}$, choose arbitrary $\mathbb{P}_{1} \in \mathcal{P}_{h}^{\kappa}$ and let $\mathbb{P}:=$ $\mathbb{P}_{1} \otimes_{t} \mathbb{P}_{2}$. Then $\mathbb{P} \in \mathcal{P}_{h}^{\kappa}$ and, by (6.4),

$$
\begin{aligned}
\mathcal{Y}_{t}^{\mathbb{P}}\left(1, g\left(x+B_{1}-B_{t}\right)\right)(\omega) & =\mathcal{Y}_{t}^{t, x, \mathbb{P}^{t, \omega}}\left(1, g\left(B_{1}^{t, x}\right)\right) \\
& =\mathcal{Y}_{t}^{t, x, \mathbb{P}_{2}}\left(1, g\left(B_{1}^{t, x}\right)\right) \quad \text { for } \mathbb{P} \text {-a.e. } \omega \in \Omega
\end{aligned}
$$

This implies that $J(t, x, \mathbb{P})=\mathcal{Y}_{t}^{t, x, \mathbb{P}_{2}}\left(1, g\left(B_{1}^{t, x}\right)\right)$ and thus $u(t, x) \leq \sup _{\mathbb{P} \in \mathcal{P}_{h}^{\kappa}} J(t, x$, $\mathbb{P})$.

(ii) We now prove that the lower-semicontinuity of $g$ implies that:

$$
(t, x) \longmapsto J(t, x, \mathbb{P}) \text { is lower-semicontinuous for any } \mathbb{P} \in \mathcal{P}_{h}^{\kappa} \text {. }
$$

which obviously implies the lower-semicontinuity of $u$ in view of (6.10).

For $(t, x) \in[0,1] \times \mathbb{R}^{d}$ and $\mathbb{P} \in \mathcal{P}_{h}^{\kappa}$, let $\left(t_{n}, x_{n}\right)_{n \geq 1}$ be a sequence in $[0,1] \times \mathbb{R}^{d}$ such that $\left(t_{n}, x_{n}\right) \longrightarrow(t, x)$. Denote, for each $n$,

$$
\begin{aligned}
& \xi_{n}:=\inf _{k \geq n} g\left(x_{k}+B_{1}-B_{t_{k}}\right), \quad f_{s}^{n}(y, z):=\inf _{k \geq n} f\left(s, x_{k}+B_{s}-B_{t_{k}}, y, z, \hat{a}_{s}\right), \\
& \xi_{\infty}:=\lim _{n \rightarrow \infty} \xi_{n}, \quad f^{\infty}:=\lim _{n \rightarrow \infty} f^{n},
\end{aligned}
$$

and, for $1 \leq n \leq \infty$, let $\left(\mathcal{Y}^{n}, \mathcal{Z}^{n}\right)$ denote the solution to the following BSDE:

$$
\mathcal{Y}_{s}^{n}=\xi_{n}-\int_{s}^{1} f_{r}^{n}\left(\mathcal{Y}_{r}^{n}, \mathcal{Z}_{r}^{n}\right) d r-\int_{s}^{1} \mathcal{Z}_{r}^{n} d B_{r}, \quad t \leq s \leq 1, \quad \mathbb{P} \text {-a.s. }
$$


By Assumptions 5.1 and 5.7, $g$ and the modulus of continuity $\rho$ of $f$ have polynomial growth in $x$. Then there exist some constants $C$ and $p$ such that

$$
\sup _{n \geq 1}\left\{\left|\xi_{n}\right|+\left|f_{t}^{n}(0,0)\right|\right\} \leq\left|\hat{f}_{r}^{0,0}\right|+C\left(\sup _{k \geq 1}\left|x_{k}\right|^{p}+\sup _{0 \leq t \leq 1}\left|B_{t}\right|^{p}\right) .
$$

Moreover, $\hat{a}$ has upper bound $\bar{a}_{\mathbb{P}}, \mathbb{P}$-a.s. then it follows from the Lipschitz conditions of $f$ that the above BSDE has a unique solution for each $n$, and

$$
\lim _{n \rightarrow \infty} \mathbb{E}^{\mathbb{P}}\left[\mathcal{Y}_{t}^{n}\right]=\mathbb{E}^{\mathbb{P}}\left[\mathcal{Y}_{t}^{\infty}\right]
$$

By the lower semi-continuity of $g$ and the uniform continuity of $f$ in $x$ in (5.3), we have $\xi_{\infty} \geq g\left(x+B_{1}-B_{t}\right)$ and $f_{s}^{\infty}(y, z)=f\left(s, x+B_{1}-B_{s}, y, z, \hat{a}_{s}\right), \mathbb{P}$-a.s. Then by the comparison principle of BSDEs one can easily see that

$$
\begin{aligned}
\varliminf_{n \rightarrow \infty} J\left(t_{n}, x_{n}, \mathbb{P}\right) & \geq \lim _{n \rightarrow \infty} \mathbb{E}^{\mathbb{P}}\left[\mathcal{Y}_{t}^{n}\right]=\mathbb{E}^{\mathbb{P}}\left[\mathcal{Y}_{t}^{\infty}\right] \\
& \geq \mathbb{E}^{\mathbb{P}}\left[\mathcal{Y}_{t}^{\mathbb{P}}\left(1, g\left(x+B_{1}-B_{t}\right)\right)\right]=J(t, x, \mathbb{P}) .
\end{aligned}
$$

This proves the lower-semicontinuity of $J$ for any fixed $\mathbb{P} \in \mathcal{P}_{h}^{\kappa}$.

We now can prove the dynamic programming principle for arbitrary stopping times.

Proof of Proposition 5.14 For any $(t, x), \mathbb{P} \in \mathcal{P}_{h}^{\kappa, t}, \mathbb{F}^{t}$-stopping time $\tau$, and any $n$, denote

$$
\tau_{n}:=\sum_{i=1}^{n} \frac{i}{n} \mathbf{1}_{\left[i-\frac{1}{n}, \frac{i}{n}\right)}(\tau)+\mathbf{1}_{\{\tau=1\}}
$$

Then $\tau_{n}$ is an $\mathbb{F}^{t}$-stopping time, $\tau_{n} \geq \tau$, and $\tau_{n} \rightarrow \tau$. By Lemma 6.4, together with Proposition 5.10, we have

$$
\mathcal{Y}_{t}^{t, x, \mathbb{P}}\left(\tau_{n}, u\left(\tau_{n}, B_{\tau_{n}}^{t, x}\right)\right) \leq u(t, x)
$$

Since $u$ is lower-semicontinuous, $\lim _{n \rightarrow \infty} u\left(\tau_{n}, B_{\tau_{n}}^{t, x}\right) \geq u\left(\tau, B_{\tau}^{t, x}\right)$. Then it follows from the comparison and the stability of BSDEs that

$$
u(t, x) \geq \mathcal{Y}_{t}^{t, x, \mathbb{P}}\left(\tau, u\left(\tau, B_{\tau}^{t, x}\right)\right)
$$

Finally, $u$ is measurable since it is lower-semicontinuous. Then (6.3) provides the opposite inequality. 


\section{Appendix}

7.1 Non-uniqueness in $\mathbb{L}^{2}\left(\mathbb{P}_{0}\right)$ of the 2BSDE (3.9)

In this section, we provide an example which shows the importance of the constraints imposed in [4] to obtain uniqueness.

Example 7.1 Consider the following two-dimensional forward SDEs:

$$
\left\{\begin{array}{l}
Y_{t}=-\int_{0}^{t} \frac{3 Y_{s}}{1-s} d s+\int_{0}^{t} \frac{X_{s}}{\sqrt{1-s}} d B_{s}, \\
X_{t}=1-\int_{0}^{t} \frac{3\left(1+c^{2}\right) X_{s}}{2 c^{2}(1-s)} d s+\int_{0}^{t} \frac{3 Y_{s}}{c \sqrt{1-s}} d B_{s},
\end{array} \mathbb{P}_{0}\right. \text {-a.s. }
$$

Clearly, (7.1) is well-posed on $[0,1)$. Denote

$$
Z_{t}:=\frac{X_{t}}{\sqrt{1-t}} ; \quad \Gamma_{t}:=\frac{3 Y_{t}}{c(1-t)} ; \quad A_{t}:=-\left(\frac{3}{2 c^{2}}+1\right) \frac{X_{t}}{(1-t)^{3 / 2}} .
$$

Then $(Y, Z, \Gamma, A)$ is a nonzero solution to $2 \mathrm{BSDE}$ (3.9).

Proof. First, applying Itô's formula one can check straightforwardly that $(Y, Z, \Gamma, A)$ satisfies the SDEs in (3.9). Notice that

$$
R_{t}:=\frac{3}{c^{2}} Y_{t}^{2}+X_{t}^{2} \text { satisfies } d R_{t}=-\frac{3 R_{t}}{1-t} d t+(\cdots) d B_{t},
$$

by Itô's formula. Since $R_{0}=1$,

$$
\mathbb{E}^{\mathbb{P}_{0}}\left[R_{t}\right]=1-3 \int_{0}^{t} \frac{\mathbb{E}^{\mathbb{P}_{0}}\left[R_{s}\right]}{1-s} d s \text { and thus } \mathbb{E}^{\mathbb{P}_{0}}\left[R_{t}\right]=(1-t)^{3}, \quad \text { for all } 0 \leq t<1 .
$$

Then one can easily see that,

$$
\sup _{0 \leq t<1} \mathbb{E}^{\mathbb{P}_{0}}\left[\left|\Gamma_{t}\right|^{2}+\left|A_{t}\right|^{2}\right] \leq C \mathbb{E}^{\mathbb{P}_{0}}\left[\frac{\left|Y_{t}\right|^{2}}{(1-t)^{2}}+\frac{\left|X_{t}\right|^{2}}{(1-t)^{3}}\right] \leq C,
$$

which, together with (3.9), also implies that

$$
\mathbb{E}^{\mathbb{P}_{0}}\left[\sup _{0 \leq t<1}\left[\left|Y_{t}\right|^{2}+\left|Z_{t}\right|^{2}\right] \leq C .\right.
$$


Finally, we prove that

$$
\lim _{t \uparrow 1} Y_{t}=0, \quad \mathbb{P}_{0} \text {-a.s. }
$$

In fact, for any $t<T<1$, by Burkholder-Davis-Gundy inequality we have

$$
\begin{aligned}
\mathbb{E}\left[\sup _{t \leq s \leq T}\left|Y_{S}\right|^{2}\right] & \leq C \mathbb{E}\left[\left|Y_{t}\right|^{2}+\int_{t}^{T} \frac{\left|Y_{s}\right|^{2}}{(1-s)^{2}} d s+\int_{t}^{T} \frac{\left|X_{s}\right|^{2}}{1-s} d s\right] \\
& \leq C\left((1-t)^{3}+\int_{t}^{T}\left((1-s)+(1-s)^{2}\right) d s\right] \leq C(1-t)^{2} .
\end{aligned}
$$

Let $T \uparrow 1$ and apply the monotone convergence Theorem, we get

$$
\mathbb{E}\left[\sup _{t \leq s<1}\left|Y_{s}\right|^{2}\right] \leq C(1-t)^{2}
$$

Then $\sup _{t \leq s<1}\left|Y_{S}\right|^{2} \downarrow 0$, as $t \uparrow 1, \mathbb{P}_{0}$-a.s. by the decrease of $\sup _{t \leq s<1}\left|Y_{s}\right|^{2}$ in $t$, and we deduce (7.2).

\subsection{Proof of Lemma 4.2}

If the a priori estimates (4.4) and (4.5) hold, then by the martingale representation property (2.5), the Lipschitz conditions (2.9), and the integrability assumption of $\hat{F}^{0}$ in (2.8), following the standard arguments one can easily show that BSDE (4.3) has a unique solution.

We now prove (4.4) and (4.5). For notational simplicity in the proof we drop the superscripts $\mathbb{P}$ in $\left(\mathcal{Y}^{\mathbb{P}}, \mathcal{Z}^{\mathbb{P}}\right)$. By the Lipschitz conditions (2.9), there exist bounded processes $\lambda, \eta$ such that

$$
\mathcal{Y}_{t}=\xi+\int_{t}^{1}\left(\hat{F}_{s}^{0}+\lambda_{s} \mathcal{Y}_{s}+\eta_{s} \hat{a}_{s}^{1 / 2} \mathcal{Z}_{s}\right) d s-\int_{t}^{1} \mathcal{Z}_{s} d B_{s}, \quad 0 \leq t \leq 1, \quad \mathbb{P} \text {-a.s. }
$$

Define $M$ by (4.10). By Itô's formula, we have:

$d\left(M_{t} \mathcal{Y}_{t}\right)=-M_{t} \hat{F}_{t}^{0} d t+M_{t}\left(\mathcal{Z}_{t}-\mathcal{Y}_{t} \eta_{t} \hat{a}_{t}^{-1 / 2}\right) d B_{t}, \quad 0 \leq t \leq 1, \quad \mathbb{P}$-a.s.

Then, using standard localization arguments if necessary:

$$
\mathcal{Y}_{t}=M_{t}^{-1} \mathbb{E}_{t}^{\mathbb{P}}\left[M_{1} \xi+\int_{t}^{1} M_{s} \hat{F}_{s}^{0} d s\right], \quad 0 \leq t \leq 1, \quad \mathbb{P} \text {-a.s. }
$$


It follows from (4.12) that, for $1<\kappa \leq 2$,

$$
\begin{aligned}
\left|\mathcal{Y}_{t}\right| & \leq \mathbb{E}_{t}^{\mathbb{P}}\left[\sup _{t \leq s \leq 1}\left(M_{t}^{-1} M_{s}\right)\left(|\xi|+\int_{t}^{1}\left|\hat{F}_{s}^{0}\right| d s\right)\right] \\
& \left.\leq C_{\kappa}\left(\mathbb{E}_{t}^{\mathbb{P}}\left[|\xi|^{\kappa}+\int_{t}^{1}\left|\hat{F}_{s}^{0}\right|^{\kappa} d s\right)\right]\right)^{1 / \kappa}, \quad 0 \leq t \leq 1, \quad \mathbb{P} \text {-a.s. }
\end{aligned}
$$

This proves (4.4).

Finally, applying Itô's formula on $\mathcal{Y}_{t}^{2}$ and following standard arguments we have

$$
\begin{aligned}
\mathbb{E}^{\mathbb{P}}\left[\int_{0}^{1}\left|\hat{a}_{t}^{1 / 2} \mathcal{Z}_{t}\right|^{2} d t\right] & \leq C \mathbb{E}^{\mathbb{P}}\left[|\xi|^{2}+\sup _{0 \leq t \leq 1}\left|\mathcal{Y}_{t}\right| \int_{0}^{1}\left|\hat{F}_{t}^{0}\right| d t\right] \\
& \leq C \mathbb{E}^{\mathbb{P}}\left[\sup _{0 \leq t \leq 1}\left|\mathcal{Y}_{t}\right|^{2}+\left(\int_{0}^{1}\left|\hat{F}_{t}^{0}\right| d t\right)^{2}\right] \\
& \leq C \mathbb{E}^{\mathbb{P}}\left[\sup _{0 \leq t \leq 1}\left|\mathcal{Y}_{t}\right|^{2}+\left(\int_{0}^{1}\left|\hat{F}_{t}^{0}\right|^{\kappa} d t\right)^{2 / \kappa}\right]
\end{aligned}
$$

This, combing with (4.4), proves (4.5).

\subsection{Proof of (4.17)}

By the definition of $\mathcal{P}_{H}^{\kappa}$, we have $\mathbb{P}=\mathbb{P}^{\alpha}, \mathbb{P}_{1}^{\prime}=\mathbb{P}^{\alpha^{1}}$, and $\mathbb{P}_{2}^{\prime}=\mathbb{P}^{\alpha^{2}}$ for $\mathbb{F}$-progressively measurable processes $\alpha, \alpha^{1}, \alpha^{2}$ taking values in $\mathbb{S}_{d}^{>0}$. Since $\mathbb{P}, \mathbb{P}_{1}^{\prime}, \mathbb{P}_{2}^{\prime} \in \mathcal{P}_{H}^{\kappa}$, by (2.8) there exist $\underline{\alpha}, \bar{\alpha}, \underline{\alpha}^{i}, \bar{\alpha}^{i} \in \mathbb{S}_{d}^{>0}$ such that

$$
\underline{\alpha} \leq \alpha \leq \bar{\alpha}, \quad \underline{\alpha}^{i} \leq \alpha^{i} \leq \bar{\alpha}^{i}, \quad d t \times d \mathbb{P}_{0} \text {-a.s. }
$$

Since $\mathbb{P}_{i}^{\prime} \in \mathcal{P}_{H}^{\kappa}(t, \mathbb{P})$, it is clear that $\alpha=\alpha^{i}, d s \times d \mathbb{P}_{0}$-a.s. on $[0, t] \times \Omega$. Recall (2.3), then

$$
\begin{aligned}
& \alpha_{s}^{*}(\omega):=\alpha_{s}(\omega) \mathbf{1}_{[0, t)}(s)+\left(\alpha_{s}^{1}(\omega) \mathbf{1}_{\left\{X^{\alpha} \in E_{1}\right\}}(\omega)\right. \\
& \left.+\alpha_{s}^{2}(\omega) \mathbf{1}_{\left\{X^{\alpha} \in E_{2}\right\}}(\omega)\right) \mathbf{1}_{[t, 1]}(s), \quad s \in[0,1]
\end{aligned}
$$

is $\mathbb{F}$-progressively measurable and satisfies:

$$
0<\underline{\alpha} \wedge \underline{\alpha}^{1} \wedge \underline{\alpha}^{2} \leq \alpha^{*} \leq \bar{\alpha} \vee \bar{\alpha}^{1} \vee \bar{\alpha}^{2}
$$


Following a line by line analogy of the proof of Claim 4.19 in [17], which in turn uses the arguments in the proof of Lemma 4.1 in [17], we see that $\mathbb{P}^{\prime}=\mathbb{P}^{\alpha^{*}} \in \overline{\mathcal{P}}_{S}$. Moreover,

$$
\begin{aligned}
\mathbb{E}^{\mathbb{P}^{\prime}}\left[\int_{0}^{1}\left|\hat{F}_{s}^{0}\right|^{2} d s\right] & =\mathbb{E}^{\mathbb{P}}\left[\int_{0}^{t}\left|\hat{F}_{s}^{0}\right|^{2} d s\right]+\mathbb{E}^{\mathbb{P}_{1}^{\prime}}\left[\int_{t}^{1}\left|\hat{F}_{s}^{0}\right|^{2} d s \mathbf{1}_{E_{1}}\right]+\mathbb{E}^{\mathbb{P}_{2}^{\prime}}\left[\int_{t}^{1}\left|\hat{F}_{s}^{0}\right|^{2} d s \mathbf{1}_{E_{2}}\right] \\
& \leq \mathbb{E}^{\mathbb{P}}\left[\int_{0}^{1}\left|\hat{F}_{s}^{0}\right|^{2} d s\right]+\mathbb{E}^{\mathbb{P}_{1}^{\prime}}\left[\int_{0}^{1}\left|\hat{F}_{s}^{0}\right|^{2} d s \mathbf{1}_{E_{1}}\right]+\mathbb{E}^{\mathbb{P}_{2}^{\prime}}\left[\int_{0}^{1}\left|\hat{F}_{s}^{0}\right|^{2} d s \mathbf{1}_{E_{2}}\right]<\infty
\end{aligned}
$$

Then $\mathbb{P}^{\prime} \in \mathcal{P}_{H}^{\kappa}$. Obviously, $\mathbb{P}^{\prime}=\mathbb{P}$ on $\mathcal{F}_{t}$. This proves that $\mathbb{P}^{\prime} \in \mathcal{P}_{H}^{\kappa}(t, \mathbb{P})$.

Acknowledgments We are grateful to Marcel Nutz for his careful reading which helped clarifying some technical points of the proofs.

\section{References}

1. Bismut, J.M.: Conjugate convex functions in optimal stochastic control. J. Math. Anal. Appl. 44, 384404 (1973)

2. Bouchard, B., Touzi, N.: Weak dynamic programming principle for viscosity solutions (2009, preprint)

3. Cheridito, P., Soner, H.M., Touzi, N.: The multi-dimensional super-replication problem under Gamma constraints. Annales de l'Institut Henri Poincaré, Série C Analyse Non-Linéaire 22, 633-666 (2005)

4. Cheridito, P., Soner, H.M., Touzi, N., Victoir, N.: Second order BSDE's and fully nonlinear PDE's. Commun. Pure Appl. Math. 60(7), 1081-1110 (2007)

5. Crandall, M.G., Ishii, H., Lions, P.L.: User's guide to viscosity solutions of second order partial differential equations. Bull. Am. Math. Soc. 27(1), 1-67 (1992)

6. Denis, L., Martini, C.: A theorectical framework for the pricing of contingent claims in the presence of model uncertainty. Ann. Appl. Probab. 16(2), 827-852 (2006)

7. Denis, L., Hu, M., Peng, S.: Function spaces and capacity related to a sublinear expectation: application to G-Brownian motion paths. Potential Anal. 34(2), 139-161 (2011)

8. El Karoui, N., Peng, S., Quenez, M.-C.: Backward stochastic differential equations in finance. Math. Finance 7, 1-71

9. Föllmer, H.: Calcul d'Ito sans probabilities. In: Seminar on Probability XV. Lecture Notes in Math., vol. 850, pp. 143-150. Springer, Berlin (1981)

10. Karandikar, R.: On pathwise stochastic integration. Stoch. Process. Appl. 57, 11-18 (1995)

11. Neveu, J.: Discrete Parameter Martingales. North-Holland, Amsterdam (1975)

12. Pardoux, E., Peng, S.: Adapted solution of a backward stochastic differential equation. Syst. Control Lett. 14, 55-61 (1990)

13. Peng, S.: G-Brownian motion and dynamic risk measure under volatility uncertainty (2007). arXiv:0711.2834v1

14. Soner, H.M., Touzi, N.: The dynamic programming equation for second order stochastic target problems. SIAM J. Control Optim. 48(4), 2344-2365 (2009)

15. Soner, H.M., Touzi, N., Zhang, J.: Quasi-sure stochastic analysis through aggregation. Electron. J. Probab. (2011, forthcoming). arXiv:1003.4431v1

16. Soner, H.M., Touzi, N., Zhang, J.: Martingale representation theorem for the $G$-expectation. Stoch. Process. Appl. 121, 265-287 (2011)

17. Soner, H.M., Touzi, N., Zhang, J.: Dual Formulation of Second Order Target Problems (2009). arXiv: 1003.6050

18. Stroock, D.W., Varadhan, S.R.S.: Multidimensional Diffusion Processes. Springer, Berlin (1979) 